\title{
Solar Process Heat in Industrial Systems- A Global Review
}

\author{
Shahjadi Hisan Farjana ${ }^{a}$, Nazmul Huda ${ }^{a, n}$, M. A. Parvez Mahmud ${ }^{a}$, R. Saidur ${ }^{b, c}$ \\ a Sustainable Energy Systems Engineering (SESE), School of Engineering, Macquarie University, Sydney, Australia \\ ${ }^{b}$ Research Centre for Nano-Materials and Energy Technology (RCNMET), School of Science and Technology, Sunway University, \\ No. 5, Jalan Universiti, Bandar Sunway, Petaling Jaya, 47500 Selangor Darul Ehsan, Malaysia \\ ${ }^{\circ}$ Department of Engineering, Lancaster University, LA1 4YW,UK
}

\begin{abstract}
In developing countries, industries and manufacturing sectors consume a major portion of the total consumption of energy, where most of the energy is used for low, medium or high temperature heat generation to be used for process applications known as process heat. The necessity to commercialize clean, cheap and efficient renewable sources of energy in industrial applications emerges from increasing concerns about greenhouse gas emissions and global warming and decreasing fossil fuel use in commercial sectors. As an abundant source of energy, solar energy technologies have proven potential. Recent research shows currently only a few industries are employing solar energy in industrial processes to generate process heat while replacing fossil fuels. Solar thermal power generation is already very well-known and getting popular in recent years while other potential applications of the concentrated heat from solar radiation are little explored. This review paper presents a detailed overview of the current potential and future aspects of involving solar industrial process heating systems in industrial applications. In order to keep pace with this emerging and fast growing sector for renewable energy applications, it is necessary to get in depth knowledge about the overall potential of industrial processes in individual industrial sector where solar process heat is currently in use and identifying industrial processes are most compatible for solar system integration depending on temperature level and the type of solar collector in use. Furthermore, the promising sectors needs to be identified for the use of solar heat using industrial processes for the integration of solar heat, so that countries with immense solar energy potential can use those technologies in future to reduce fossil fuel consumption and develop sustainable industrial systems. This paper presents a comprehensive review of the potential industrial processes that can adopt solar process heating systems and thus driving towards sustainable production in industries.
\end{abstract}

Contents

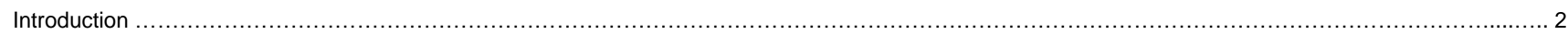

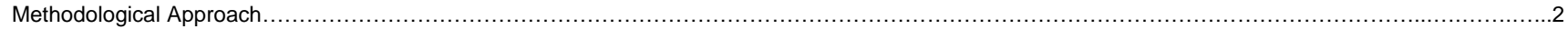

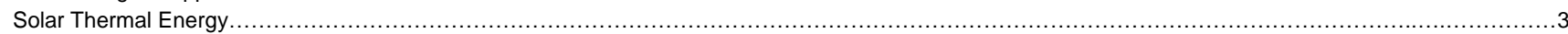

Solar Industrial Process Heating Systems.

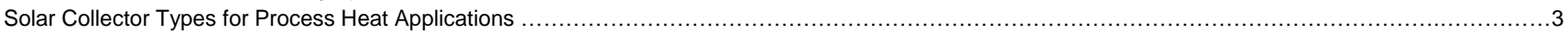

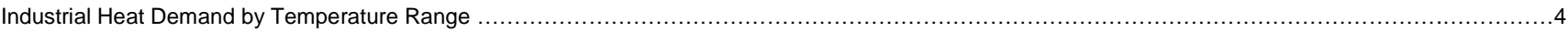

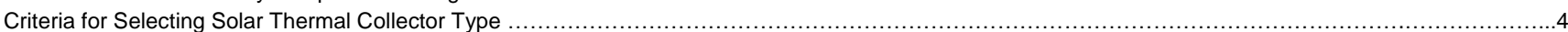

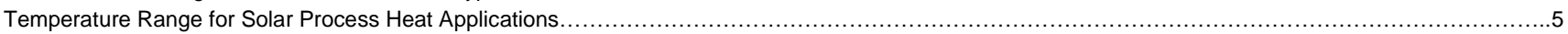

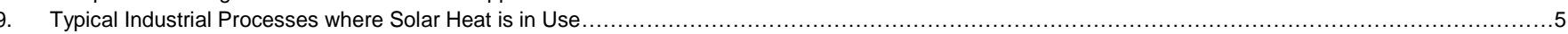

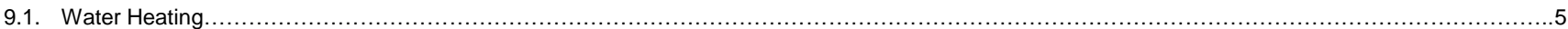

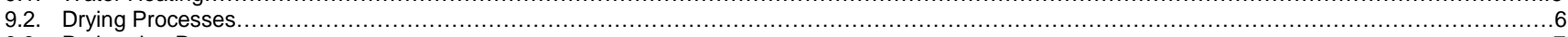

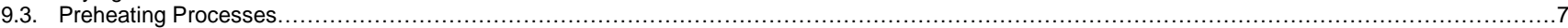

9.4. Steam Heating .

9.5. Pasteurization, Sterilization Process

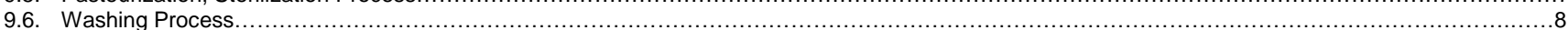

10. Currently used Solar Industrial Process Heat Systems …...........9

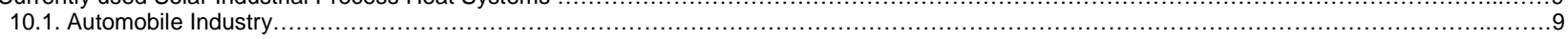

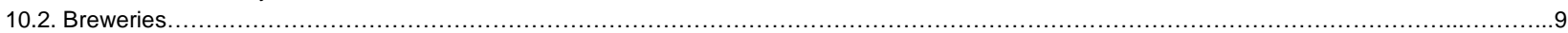

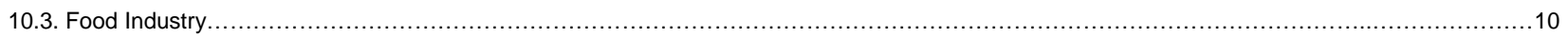

${ }^{\mathrm{n}}$ Corresponding author. Tel.: +61298509598
E-mail address: nazmul.huda@mq.edu.au (Dr. Nazmul Huda) 


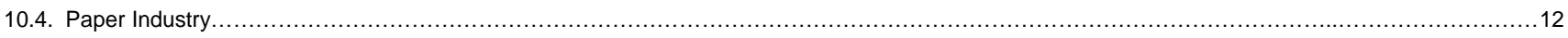

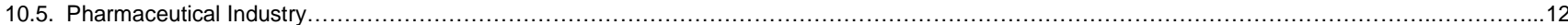

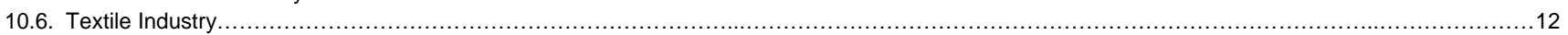

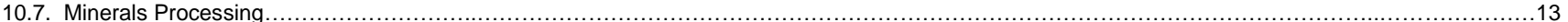

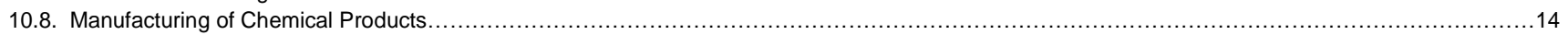

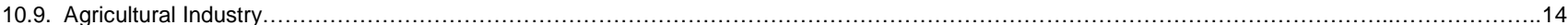

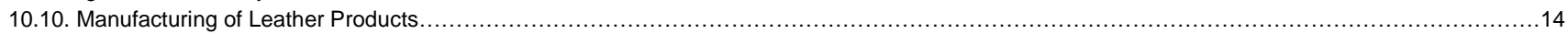

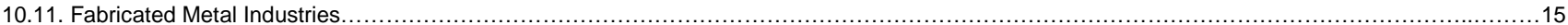

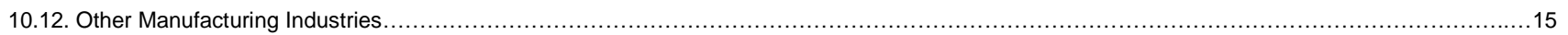

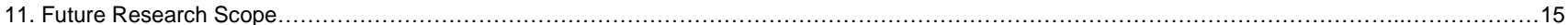

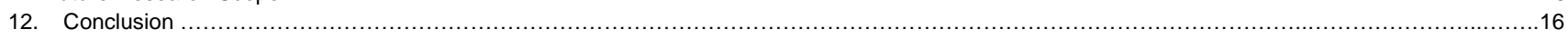

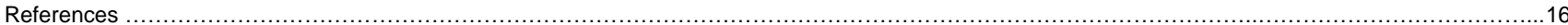

\section{Introduction}

Recent trends of energy production and consumption in manufacturing industries are notably unsustainable due to rapidly increasing greenhouse gas emissions. Developing countries throughout the world are trying to industrialize their own economy to create new opportunities for the young generation to address their own country as a developed one. For mitigating poverty through sustainable development, every developing country is following their own energy policy for employing environmentally friendly energy specifically in the industrial sectors $[4,7,12,13,99]$.

As a renewable and abundant source, solar energy systems are future sustainable solutions for industrial consumers although, some places have inadequate solar energy resource where solar industrial process heating may not be feasible, but many other places receive abundant solar radiation and implementing such systems will drive the industries towards sustainable zero carbon production future. Industrial processes are mainly dependent on either electricity or fossil fuels to supply industrial process heat. As the non-renewable energy sources are going to be finished in future and considering all the drawbacks like GHG emissions, demand for renewable energy sources is imminent. The major drawback which hinders the scope for small and medium scale industries to integrate renewable energy systems in their production processes is the high capital cost while mostly the importance goes for ensuring continued supply of process heat from solar energy systems $[7,12,13,100]$.

Industrialized and developed regions in Europe, Asia and North America are successfully using solar thermal systems in industrial processes. Solar thermal energy for electricity generation is also becoming popular in different parts of the world. Feasibility of commissioning and running such systems are heavily dependent on radiation intensity. If the regional and seasonal variation is not significant, then a supplementary energy storage system may not be necessary depending on the type of application. Where seasonal variation is quite dominant where reduced radiation available in winter for a few months, energy storage systems may improve the overall performance of the system.
This review paper discussed the fundamentals of solar thermal energy and solar collectors, and the temperature range for industrial applications where process heat is in use. According to solar industrial process heating (SHIP) plants database information, promising industrial sectors are identified and further explained with current literature and plant installation information. The most widely used industrial processes with solar thermal applications are thus identified and reviewed. This review aims to identify the existing potential of solar industrial process heating systems in industrial sectors, where to integrate solar industrial process heating systems, which collectors are suitable for specific processes depending on temperature requirements. The authors systematically analyzes existing solar industrial process heating plants throughout the world, thus identify 11 key industrial sectors, mostly developed 6 processes. In these identified and integrated industrial sectors and processes are categorized through suitable solar collector type and process heating temperature ranges. This paper also discusses the future potential of solar industrial process heating systems.

Section 1 gives an overview of the importance of solar process heat for industrial sectors, and the objectives of this review paper. Section 2 discusses the present literature, trying to identify the potential sectors for solar industrial process heating systems. Section 3 and Section 4 gave an insight into solar thermal energy and solar industrial process heat. Section 5 illustrates the most widely used solar collector types for process heating systems. The process heat requirements by temperature range are discussed in section 6 . Then section 7 explains the criteria for selecting a suitable solar collector for an integrated solar process heating system. Section 8 worked on temperature range of solar process heat applications, followed by Section 9 where most common solar driven industrial process stages are discussed through current potential, plants in operation and existing literature. Section $\mathbf{1 0}$ described a range of industries where solar process heat is in use, how they are employing solar process heat, where they are employing it and how it is performing. Finally Section 11 and Section 12 analyze and compare existing potential research and reviews throughout all industrial sectors to find gaps in the gap of research and also the gap of solar process heating system integration in industries. 


\section{Methodological Approach}

There are very few research papers which try to identify potential industrial sectors for integration of solar heat in industrial processes and none of them analyze the potential industrial sectors in respect of existing SHIP plants globally to determine the potentials for others which are feasible and already in practise. This review paper is the first one to do such an analysis in SHIP.

Lauterbach et al. studied and reviewed the potential in the field of solar industrial process heat integrated with suitable industrial processes. The annual specific energy gain is determined using TRNSYS simulation depending on temperature level, location and solar collector type [44]. In another work, Lauterbach et al. methodically analyzed an installed solar process heating system based on simulation and compared it with a validated model [42]. Weiss et al. reviewed the solar industrial process heating potential among countries for medium temperature collectors and sought a solution for SHIP integration problem [94]. Schnitzer et al. applied a newly developed investigation tool to investigate industrial energy systems and heat integration feasibilities through an Austrian dairy industry case study [77]. IEA SHC task $\mathbf{3 3}$ and SolarPaces Task IV: Solar Heat for Industrial Processes identifies and discusses potential industrial sectors for SHIP integration [36]. Mekhilef et al. reviewed solar energy utilization by industrial applications thus identified potential industries [53]. Norton illustrated the industrial applications of solar heat such as solar water heating system, solar drying system, solar furnaces, green houses, heating and ventilation systems, solar cooking, solar desalination and solar refrigeration [63, 64]. Vajen et al. outlined solar heat integration potential at supply level and process level in Europe, Germany and worldwide [91]. Taibi et al. reviewed and discussed renewable energy potential in industrial sectors such as biomass and low temperature solar process heat [89]. Schmitt performed several comprehensive studies to show the utilized process installations in food and beverage industries. He also developed a classification mechanism for SHIP integration [76]. Modi et al. reviewed solar driven heat and power generation systems [54]. Pietruschka et al. reported on the fundamental design, process system layout, operating conditions and industrial processes of three large scale solar process heat installations [69]. Calderoni et al. studied the feasibility analysis of integration of solar process heating systems in industrial processes in Tunisia followed by an economic analysis [9]. Montes et al. presented the parabolic trough solar collector based process heating system and their design results [56]. Frein et al. studies and presents the design procedure and analysis basics required for integrating a solar thermal plant into an industrial system [27]. Schramm et al. presents a new concept of solar process heat integration system based on solar tanks through solar tank volume simulation results [78]. Larcher et al. experimentally investigated the parabolic trough solar collector based integrated solar process heating system [41]. Kalogirou presented simulated results of the parabolic trough collector based solar process heating system performance located on Cyprus [38]. Esen experimentally investigated and presented the thermal performance of vacuum tube collector based solar cooker under varied refrigerant conditions [21]. Silva et al. developed an optimization method for parabolic trough solar collector based solar industrial process heating systems. In another research work Silva et al. also conducted uncertainly and sensitivity analysis for parabolic trough collector based steam generation plant [83-85]. Coccia et al. designed and experimentally tested a prototype model of a parabolic trough solar collector for process heating application [11].
Naik et al. reviewed all kinds of medium temperature application areas of concentrated solar thermal technologies in India [62].

This review work analyzes existing solar industrial process heating systems throughout industries over the world. These industries and their respective process systems are analyzed and explained based on their solar collector type, installed thermal capacity and expected temperature range. Existing research and review are also studied by industrial category. Thereby the more common industrial process stages integrated with solar systems are identified and presented in this paper. These industrial process stages are elaborated with their industrial category, country data, solar collector and temperature range specifications. In a later part, potential industrial sectors are identified and discussed to find the integration problems of solar industrial process heating systems and future research scope.

In this review, all industrial sectors are analyzed based on their respective process operations where solar heat is already in use, and also classified based on process operation and temperature range. The dominant industrial sector where solar process heating system is widely in use is food and beverage industries, comprising water heating, washing, pasteurization, cooking, drying etc. Then another sector that is textile industries where cleaning, drying, washing, fixing and pressing are the major operations utilizing solar process heat. The temperature ranges required for these process operations are also identified and reviewed to locate the potential sectors which are currently consuming a significant amount of fossil fuels to run the process operations but where the low range temperature can be easily achieved by using solar process heat. Also, this review paper will be of great help for different countries where solar heat is found in abundance but is not in utilization for industrial operations. Industrial authorities can easily get an overview from this paper where to utilize solar process heat and what can be achieved from this. Future research scopes in the field of solar industrial process heat and its impact on greenhouse gas emissions and sustainable development through the lifecycle assessment are also outlined in the last section of the paper.

\section{Solar Thermal Energy}

In simple words, while we get the energy from heat conversion gained from solar irradiation, is termed as solar thermal energy. Like other renewable energy systems, solar thermal energy can replace the fossil fuels in industrial systems. The energy conversion systems employ a variety of solar thermal collectors along with concentrators which accumulate and deliver solar radiation for process heat generation to be used in commercial or industrial plants. The type of solar collector, working fluid, installation parameters, heat exchanger specifications need to be considered for the specific process operation. However, the most important factor is solar energy could not be available to supply process heat in the system for 24 hours. Supplementary process heating systems should be equipped using phase change materials (PCM) or molten salts [86-88,97-102].

\section{Solar Industrial Process Heating Systems}

Of the global final energy demand, electricity accounts for around $17 \%$ low temperature heat applications stand for $44 \%$, whereas high temperature industrial process heat occupies $10 \%$. Solar energy has a long history of use in the residential building sectors. The industrial and manufacturing sectors are well suited to solar thermal technology 
and its application only because of the volume of energy required for process heating systems in integration. For example, the major heat requirement is for processes like different types of drying, cleaning, washing, water heating, pasteurization and sterilization and so many food processing applications. The temperature level required for these industrial processes is similar for almost all applications being below $250^{\circ} \mathrm{C}$ [86-88, 97-102].

\section{Solar Collector Types for Process Heat Applications}

\section{Flat-Plate Solar Collector}

This accumulates heat energy from incident radiation while the absorber plate absorbs and then transmits it to the working fluid, for example heat transfer oil , air, water, or aqueous glycol solution. Flatplate collectors are popular for high absorbance efficiency of the incident radiation with good thermal conductivity and low emittance. During plant operation they provide thermal stability of the temperatures encountered. Flat-plate collectors are lighter, more durable and with cheaper capital cost. There are pipes of an isolated box where working fluids circulate to reduce heat losses.

\section{Evacuated-Tube Solar Collector}

In an evacuated-tube collector, a separate cylindrical glass envelope is provided which prevents the absorber plate from heat loss due to convection. The collector selection is dependent upon the temperature requirement of the industrial process.

\section{Unglazed Collector}

These are used for certain low-temperature applications, for example, for recovering salt from agricultural drainage water.

\section{Vacuum-Tube Collector}

In vacuum-tube collectors, pipes are placed inside vacuum glass tubes through which water circulates inside pipes. In comparison with flatplate collectors, higher temperature range can be obtained and heat losses can be reduced more effectively.

\section{Fresnel Collector or Parabolic Trough Collectors}

The irradiation is first incidents on the reflective surfaces and then concentrated onto a solar absorber. Here, high temperature ranges above $400^{\circ} \mathrm{C}$ can be achieved. Concentrating collectors continually track the sunshine over the whole day and over the whole year. Primary mirrors are there to track the direct irradiation from sunlight over the time and further concentrate the sunlight onto the solar absorber tube. Inside the absorber tube, a heat transfer fluid (pressurized water, steam or thermal oil) circulates to provide thermal energy to the target process.

Table 1 represents the types of solar collectors based on their key features, concentration ratio for direct isolation and indicative temperature.

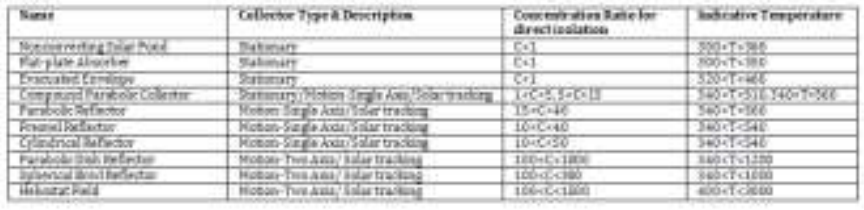

Table 1: Classification of solar collectors.

\section{Industrial Heat Demand by Temperature Range}

The analysis of the performed review studies will be useful for the industrial process heat requirement depending on the temperature ranges for many countries, which are not widely available. The analysis presented in this review paper will be useful to overcome this lack of information. However, it should be mentioned that even the industrial processes consume medium-temperature heat by using steam as a medium of working fluid; lower working temperatures would be sufficient for that purpose. Moreover, to calculate the efficiency of the integration of a solar thermal system into a manufacturing process, first the actual temperature required by the process itself should be assessed properly and should not be simply the heat carrier temperature in use. Such an approach should be followed for lowering the process energy consumption.

Table 2 represents the major industrial sectors and their application areas where low-temperature process heat is required, categorized based on process stage and temperature range. The datasets are collected from several published books and journal resources.

\begin{tabular}{|c|c|c|}
\hline Sector & Process & Temperature Range $\left({ }^{\circ} \mathrm{C}\right)$ \\
\hline \multirow[t]{5}{*}{ Chemicals } & Biochemical reaction & $20 \sim 60$ \\
\hline & Distillation & $100 \sim 200$ \\
\hline & Compression & $105 \sim 165$ \\
\hline & Cooking & $80 \sim 100$ \\
\hline & Thickening & $110 \sim 130$ \\
\hline \multirow[t]{11}{*}{ Foods \& beverages } & Blanching & $60 \sim 100$ \\
\hline & Scalding & $45 \sim 90$ \\
\hline & Evaporating & $40 \sim 130$ \\
\hline & Cooking & $70 \sim 120$ \\
\hline & Pasteurization & $60 \sim 145$ \\
\hline & Smoking & $20 \sim 85$ \\
\hline & Cleaning & $60 \sim 90$ \\
\hline & Sterilization & $100 \sim 140$ \\
\hline & Tempering & $40 \sim 80$ \\
\hline & Drying & $40 \sim 200$ \\
\hline & Washing & $30 \sim 80$ \\
\hline \multirow[t]{4}{*}{ Paper } & Bleaching & $40 \sim 150$ \\
\hline & De-inking & $50 \sim 70$ \\
\hline & Cooking & $110 \sim 180$ \\
\hline & Drying & $95 \sim 200$ \\
\hline \multirow[t]{7}{*}{ Fabricated Metal } & Pickling & $40 \sim 150$ \\
\hline & Chromaiing & $20 \sim 75$ \\
\hline & Degreasing & $20 \sim 100$ \\
\hline & Electroplating & $30 \sim 95$ \\
\hline & Phosphating & $35 \sim 95$ \\
\hline & Purging & $40 \sim 70$ \\
\hline & Drying & $60 \sim 200$ \\
\hline \multirow[t]{2}{*}{ Rubber \& Plastic } & Drying & $50 \sim 150$ \\
\hline & Preheating & $50 \sim 70$ \\
\hline \multirow{2}{*}{$\begin{array}{l}\text { Machinery \& } \\
\text { Equipment }\end{array}$} & Surface treatment & $20 \sim 120$ \\
\hline & Cleaning & $40 \sim 90$ \\
\hline \multirow[t]{6}{*}{ Textiles } & Bleaching & $40 \sim 100$ \\
\hline & Coloring & $40 \sim 130$ \\
\hline & Drying & $60 \sim 90$ \\
\hline & Washing & $50 \sim 100$ \\
\hline & Fixing & $160 \sim 180$ \\
\hline & Pressing & $80 \sim 100$ \\
\hline \multirow[t]{4}{*}{ Wood } & Steaming & $70 \sim 90$ \\
\hline & Pickling & $40 \sim 70$ \\
\hline & Compression & $120 \sim 170$ \\
\hline & Cooking & $80 \sim 90$ \\
\hline
\end{tabular}




\begin{tabular}{|c|c|c|}
\hline & Drying & $40 \sim 150$ \\
\hline \multirow[t]{5}{*}{ Dairy } & Pressurization & $60-80$ \\
\hline & Sterilization & $100-120$ \\
\hline & Drying & $120-180$ \\
\hline & Concentrates & $60-80$ \\
\hline & Boiler feed water & $60-90$ \\
\hline \multirow[t]{4}{*}{ Tinned food } & Sterilization & $110-120$ \\
\hline & Pasteurization & $60-80$ \\
\hline & Cooking & $60-90$ \\
\hline & Bleaching & $60-90$ \\
\hline \multirow[t]{3}{*}{ Meat } & Washing, & $60-90$ \\
\hline & Sterilization & $60-90$ \\
\hline & Cooking & $90-100$ \\
\hline $\begin{array}{l}\text { Flour \& By- } \\
\text { products }\end{array}$ & Sterilization & $60 \sim 80$ \\
\hline \multirow[t]{4}{*}{ Timber By-products } & Thermo diffusion beams & $80 \sim 100$ \\
\hline & Drying & $60 \sim 100$ \\
\hline & Pre-heating water & $60 \sim 90$ \\
\hline & Preparation pulp & $120 \sim 170$ \\
\hline Bricks \& Blocks & Curing & $60 \sim 140$ \\
\hline \multirow[t]{6}{*}{ Plastics } & Preparation & $120 \sim 140$ \\
\hline & Distillation & $140 \sim 150$ \\
\hline & Separation & $200 \sim 220$ \\
\hline & Extension & $140 \sim 160$ \\
\hline & Drying & $180 \sim 200$ \\
\hline & Blending & $120 \sim 140$ \\
\hline \multirow[t]{3}{*}{ Automobile } & Water heating & $\sim 90$ \\
\hline & Cleaning & $\sim 120$ \\
\hline & Other processes & $\sim 50$ \\
\hline Pharmacy & Different processes & $7 \sim 180$ \\
\hline \multirow[t]{3}{*}{ Mine } & Cleaning & $\sim 60$ \\
\hline & Electro-Winning & $\sim 50$ \\
\hline & Other processes & $\sim 80$ \\
\hline \multirow{2}{*}{ Agriculture } & Drying & $\sim 80$ \\
\hline & Water Heating & $\sim 90$ \\
\hline \multirow[t]{2}{*}{ Leather } & Retanning & $\sim 80$ \\
\hline & Other processes & $\sim 90$ \\
\hline \multirow[t]{2}{*}{ Metal } & Heating & $\sim 180$ \\
\hline & Washing & $\sim 160$ \\
\hline
\end{tabular}

Table 2: Solar Thermal Energy for Industrial Uses.

\section{Criteria for Selecting Solar Thermal Collector Type}

There are some required aspects which are especially relevant and need to be considered to identify the optimal solar thermal collector technology such as space efficiency, temperature control and integration. Solar energy is affluently available where comparatively large areas are required for the collection of solar energy. Every industry is energy intensive where unused space is scarce in industrial areas. So, to use solar process heat in industries, solar-collector technology should have space efficiency with roof top installations. Another point is industrial processes are often very sensitive to temperature ranges. Definitely the solar collector systems should provide accurate temperatures according to the specific demand. Most industries use heat-carrier fluid which can supply heat to different processes. Solar process heating systems are integrated with conventional heating systems.

For integration of a solar thermal energy system into a conventional system, there could be three main methods.

1. Pre-heating of water where heat energy is required for evaporation. However, the condensate can return a high temperature so the efficiency of the non-concentrating collector is reduced.

2. Integration on the distribution level where high temperatures (mostly steam) are required so only concentrating solar collectors can be used. This provides the greatest flexibility as it is not linked to a specific process.

3. Another important way to integrate solar thermal energy is direct coupling with a specific process. But when the process is changed or stopped, it can be challenging to use the energy which is rather inflexible $[1,3,4,6,8,9]$.

\section{Temperature Range for Solar Process Heat Applications}

Solar heat can be integrated at the process level on heat supply level of an industrial company. At the supply level, preheating boiler feed water with the heating of a hot water circuit or production of direct steam are the main applications. These supply level applications require a high temperature which may turn as an unfavorable climate conditions. Depending on the size of the system and the industrial sector, other characteristics such as processes with integrated solar heating system were documented. 36 or $51 \%$ of installed solar heating systems were used for water heating or washing processes, $14 \%$ systems were integrated in "heating of baths/vessels", $6 \%$ for different drying processes and $29 \%$ for other processes like car washing facilities, etc. (Task33, 2006). The temperature level required for an industrial process is crucial for the feasibility assessment, as the annual energy yield depends strongly on provided temperature. Numerous processes can be found at temperatures below $40^{\circ} \mathrm{C}$, and $40^{\circ} \mathrm{C}$ to $60^{\circ} \mathrm{C}$. The industrial processes within the temperature range of $60^{\circ} \mathrm{C}$ to $100^{\circ} \mathrm{C}$, are of high importance as well. Figure 1 demonstrates the importance of the above mentioned temperature ranges (Task33, 2006) $[12,13,16,25,26,31,32,35]$.

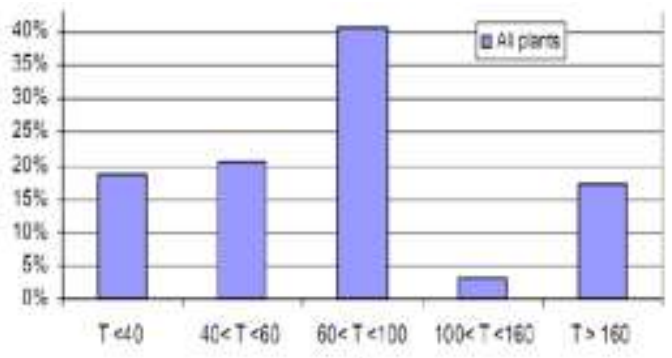

Fig. 1: Temperature level of the supported processes of existing solar heating systems (Task33, 2006) [36]

\section{Typical Industrial Processes where Solar Heat is in Use}

The analysis of industrial processes cannot be generalized or categorized because of the conditions, which vary too widely, for example, in food industry purification or bottle cleaning plants. Several unit operations operating in various processes and industrial sectors are given below.

\subsection{Water Heating}

A typical water heating process with conventional energy sources runs with smaller equipment like heat exchangers. To integrate a solar energy system, design should be focused to operate at the lowest temperature levels after increasing collector efficiency. Solar water heating (SWH) applications constitute the major part of industrial solar thermal applications, with cost effectiveness of all the currently available solar thermal technologies.

Morrison reviewed solar water heater product configurations and their markets in Australia [57]. Esen et al. research on the performance of two-phase solar collectors used in solar water heaters with different refrigerants [22]. Buker et al. reviewed past and present research work conducted on solar assisted heat pump systems used in lowtemperature solar water heaters [8]. Gautam et al. reviewed the 
basics of thus followed by the future scenario of solar water heating systems [29].

Table 3 illustrates the main industrial application areas where solar water heating system is already in use, their key features, solar collector information and also operational temperature data.

\begin{tabular}{|c|c|c|c|c|c|}
\hline Industry & Country & Name & $\begin{array}{l}\text { Industrial } \\
\text { Operation }\end{array}$ & $\begin{array}{l}\text { Solar } \\
\text { Collector }\end{array}$ & $\begin{array}{l}\text { Temperat } \\
\text { ure }\left({ }^{\circ} \mathrm{C}\right)\end{array}$ \\
\hline Automobile & $\begin{array}{l}\text { South } \\
\text { Africa }\end{array}$ & $\begin{array}{l}\text { BMW } \\
\text { Manufa } \\
\text { cturing }\end{array}$ & $\begin{array}{l}\text { Paint } \\
\text { application } \\
\text { process }\end{array}$ & $\begin{array}{l}\text { Evacuated } \\
\text { tube } \\
\text { collector }\end{array}$ & $\sim 90$ \\
\hline \multirow[t]{4}{*}{ Breweries } & USA & $\begin{array}{l}\text { Milwauk } \\
\text { ee } \\
\text { Brewing } \\
\text { Co. }\end{array}$ & Consumption & $\begin{array}{l}\text { Flat plate } \\
\text { collector }\end{array}$ & \\
\hline & Germany & $\begin{array}{l}\text { Hutt } \\
\text { Brewery }\end{array}$ & $\begin{array}{l}\text { Heating the } \\
\text { brewing } \\
\text { water }\end{array}$ & $\begin{array}{l}\text { Flat plate } \\
\text { collector }\end{array}$ & $40 \sim 95$ \\
\hline & China & $\begin{array}{l}\text { Chongqi } \\
\text { ng } \\
\text { Beverag } \\
\text { e }\end{array}$ & $\begin{array}{l}\text { Heating for } \\
\text { warehouse }\end{array}$ & $\begin{array}{l}\text { Evacuated } \\
\text { tube } \\
\text { collector }\end{array}$ & \\
\hline & $\begin{array}{l}\text { Saudi } \\
\text { Arabia }\end{array}$ & $\begin{array}{l}\text { Nestle } \\
\text { Waters }\end{array}$ & $\begin{array}{l}\text { Bottle } \\
\text { washing }\end{array}$ & $\begin{array}{l}\text { Flat plate } \\
\text { collector }\end{array}$ & \\
\hline \multirow[t]{10}{*}{ Food } & Mexico & $\begin{array}{l}\text { Aviary } \\
\text { Food } \\
\text { Pellet El } \\
\text { Paisa } \\
\end{array}$ & $\begin{array}{l}\text { Heating } \\
\text { water }\end{array}$ & $\begin{array}{l}\text { Parallel } \\
\text { trough } \\
\text { collector }\end{array}$ & $\sim 95$ \\
\hline & \multirow[t]{2}{*}{ Greece } & $\begin{array}{l}\text { Alpino } \\
\text { S.A. }\end{array}$ & $\begin{array}{l}\text { Hot water } \\
\text { preheating in } \\
\text { steam boiler }\end{array}$ & $\begin{array}{l}\text { Flat plate } \\
\text { collector }\end{array}$ & $20 \sim 70$ \\
\hline & & & $\begin{array}{l}\text { Cleaning } \\
\text { equipment }\end{array}$ & $\begin{array}{l}\text { Other or } \\
\text { various } \\
\text { collectors }\end{array}$ & $20 \sim 90$ \\
\hline & \multirow[t]{2}{*}{ Austria } & $\begin{array}{l}\text { Futtermi } \\
\text { ttel Fruit } \\
\text { Juice }\end{array}$ & $\begin{array}{l}\text { Makeup } \\
\text { water }\end{array}$ & $\begin{array}{l}\text { Flat plate } \\
\text { collector }\end{array}$ & \\
\hline & & $\begin{array}{l}\text { Mogunti } \\
\text { a Spice } \\
\text { Making }\end{array}$ & $\begin{array}{l}\text { Cleaning and } \\
\text { production } \\
\text { process }\end{array}$ & $\begin{array}{l}\text { Flat plate } \\
\text { collector }\end{array}$ & \\
\hline & Portugal & $\begin{array}{l}\text { Knoww } \\
\text { Best } \\
\text { Foods } \\
\text { S.A. }\end{array}$ & $\begin{array}{l}\text { Tools } \\
\text { washing }\end{array}$ & $\begin{array}{l}\text { Flat plate } \\
\text { collector }\end{array}$ & $40 \sim 45$ \\
\hline & Spain & $\begin{array}{l}\text { Montes } \\
\text { ano- } \\
\text { canary } \\
\text { islands }\end{array}$ & $\begin{array}{l}\text { Wash meat } \\
\text { derivatives }\end{array}$ & $\begin{array}{l}\text { Flat plate } \\
\text { collector }\end{array}$ & $40 \sim 60$ \\
\hline & $\begin{array}{l}\text { Czech } \\
\text { Republic }\end{array}$ & $\begin{array}{l}\text { PETA } \\
\text { Bohemi } \\
\text { a } \\
\text { Pekarna } \\
\text { Sobesla } \\
\text { v }\end{array}$ & Bakery & $\begin{array}{l}\text { Flat plate } \\
\text { collector }\end{array}$ & $10 \sim 90$ \\
\hline & $\begin{array}{l}\text { Netherlan } \\
\text { ds }\end{array}$ & $\begin{array}{l}\text { Perfetti } \\
\text { van } \\
\text { melle }\end{array}$ & $\begin{array}{l}\text { Preheating of } \\
\text { hot water } \\
\text { and process } \\
\text { heat }\end{array}$ & $\begin{array}{l}\text { Flat plate } \\
\text { collector }\end{array}$ & \\
\hline & Jordan & $\begin{array}{l}\text { Seniora } \\
\text { Food } \\
\text { Industri } \\
\text { es }\end{array}$ & $\begin{array}{l}\text { Degreasing } \\
\& \quad \text { cleaning } \\
\text { requirements }\end{array}$ & $\begin{array}{l}\text { Evacuated } \\
\text { tube } \\
\text { collector }\end{array}$ & \\
\hline \multirow[t]{4}{*}{ Textile } & USA & $\begin{array}{l}\text { Acme } \\
\text { McCrary }\end{array}$ & $\begin{array}{l}\text { Textile drying } \\
\text { process }\end{array}$ & $\begin{array}{l}\text { Flat plate } \\
\text { collector }\end{array}$ & \\
\hline & \multirow[t]{2}{*}{ Greece } & $\begin{array}{l}\text { Allegro } \\
\text { S.A. } \\
\text { Children } \\
\text { s' } \\
\text { clothing } \\
\text { manufa } \\
\text { cturer }\end{array}$ & $\begin{array}{l}\text { Washing } \\
\text { machines }\end{array}$ & $\begin{array}{l}\text { Flat plate } \\
\text { collector }\end{array}$ & $33 \sim 60$ \\
\hline & & $\begin{array}{l}\text { Kastrina } \\
\text { giannis } \\
\text { S.A. }\end{array}$ & $\begin{array}{l}\text { Dyeing } \\
\text { machines }\end{array}$ & $\begin{array}{l}\text { Flat plate } \\
\text { collector }\end{array}$ & $40 \sim 90$ \\
\hline & China & $\begin{array}{l}\text { Daly } \\
\text { Textile }\end{array}$ & $\begin{array}{l}\text { Dyeing } \\
\text { process }\end{array}$ & $\begin{array}{l}\text { Flat plate } \\
\text { collector }\end{array}$ & $\sim 55$ \\
\hline
\end{tabular}

\begin{tabular}{|c|c|c|c|c|c|}
\hline & India & $\begin{array}{l}\text { Sharma } \\
\mathrm{n} \\
\text { Shawls }\end{array}$ & $\begin{array}{l}\text { Dyeing, } \\
\text { bleaching \& } \\
\text { washing } \\
\text { garments }\end{array}$ & $\begin{array}{l}\text { Flat plate } \\
\text { collector }\end{array}$ & $\sim 100$ \\
\hline \multirow[t]{2}{*}{ Mining } & \multirow[t]{2}{*}{ Germany } & $\begin{array}{l}\text { Schiffer } \\
\text { Gmbh \& } \\
\text { Co KG }\end{array}$ & $\begin{array}{l}\text { Galvanic } \\
\text { baths }\end{array}$ & $\begin{array}{l}\text { Evacuated } \\
\text { tube } \\
\text { collector }\end{array}$ & $40 \sim 70$ \\
\hline & & $\begin{array}{l}\text { Steinba } \\
\text { ch und } \\
\text { Vallman } \\
n\end{array}$ & $\begin{array}{l}\text { Galvanic } \\
\text { baths }\end{array}$ & $\begin{array}{l}\text { Evacuated } \\
\text { tube } \\
\text { collector }\end{array}$ & $60 \sim 80$ \\
\hline Chemical & India & $\begin{array}{l}\text { L'oreal } \\
\text { Punne }\end{array}$ & $\begin{array}{l}\text { Cleaning } \\
\text { process }\end{array}$ & $\begin{array}{l}\text { Flat plate } \\
\text { collector }\end{array}$ & $\sim 55$ \\
\hline \multirow[t]{3}{*}{ Agriculture } & Spain & $\begin{array}{l}\text { Acuinov } \\
\text { a } \\
\text { Andaluc } \\
\text { ia S.A. }\end{array}$ & $\begin{array}{l}\text { Heating } \\
\text { Water }\end{array}$ & $\begin{array}{l}\text { Flat plate } \\
\text { collector }\end{array}$ & $23 \sim 26$ \\
\hline & China & $\begin{array}{l}\text { Hongxin } \\
\text { Aquacul } \\
\text { ture }\end{array}$ & $\begin{array}{l}\text { Water } \\
\text { Heating }\end{array}$ & $\begin{array}{l}\text { Evacuated } \\
\text { tube } \\
\text { collector }\end{array}$ & \\
\hline & Germany & Woltow & $\begin{array}{l}\text { Water } \\
\text { Heating }\end{array}$ & $\begin{array}{l}\text { Parallel } \\
\text { trough } \\
\text { collector }\end{array}$ & \\
\hline Leather & Austria & $\begin{array}{l}\text { Gerbere } \\
\text { i } \\
\text { Kolbling } \\
\text { er }\end{array}$ & $\begin{array}{l}\text { The wet } \\
\text { process of } \\
\text { leather } \\
\text { retanning }\end{array}$ & $\begin{array}{l}\text { Flat plate } \\
\text { collector }\end{array}$ & \\
\hline $\begin{array}{l}\text { Fabricated } \\
\text { Metal }\end{array}$ & India & $\begin{array}{l}\text { SKF } \\
\text { Technol } \\
\text { ogies } \\
\text { Mysore } \\
\end{array}$ & Circulation & $\begin{array}{l}\text { Parallel } \\
\text { trough } \\
\text { collector }\end{array}$ & $\sim 95$ \\
\hline $\begin{array}{l}\text { Wearing } \\
\text { Apparel }\end{array}$ & India & $\begin{array}{l}\text { Chelsea } \\
\text { Jeans }\end{array}$ & $\begin{array}{l}\text { Dyeing } \\
\text { process }\end{array}$ & $\begin{array}{l}\text { Flat plate } \\
\text { collector }\end{array}$ & \\
\hline $\begin{array}{l}\text { Computer, } \\
\text { electronic \& } \\
\text { optical } \\
\text { products }\end{array}$ & Sweden & $\begin{array}{l}\text { Bosman } \\
\text { S } \\
\text { Lackerin } \\
\mathrm{g}\end{array}$ & $\begin{array}{l}\text { Chemical } \\
\text { baths }\end{array}$ & $\begin{array}{l}\text { Parallel } \\
\text { trough } \\
\text { collector }\end{array}$ & $\sim 160$ \\
\hline \multirow[t]{2}{*}{ Machinery } & \multirow[t]{2}{*}{ Austria } & $\begin{array}{l}\text { Hoval } \\
\text { Marchtr } \\
\text { enk }\end{array}$ & $\begin{array}{l}\text { Powder } \\
\text { coating }\end{array}$ & $\begin{array}{l}\text { Flat plate } \\
\text { collector }\end{array}$ & \\
\hline & & $\begin{array}{l}\text { Kral } \\
\text { Pump } \\
\text { Factory }\end{array}$ & $\begin{array}{l}\text { Space } \\
\text { heating }\end{array}$ & $\begin{array}{l}\text { Evacuated } \\
\text { tube } \\
\text { collector }\end{array}$ & \\
\hline
\end{tabular}

Table 3: Industries working with solar water heating process [101].

\subsection{Drying processes}

In drying processes, moisture can be eliminated from a material as a primary condition. Some other drying processes are involved in drying food, plants, fruits, textiles and mineral materials, washing and varnishing. By preheating with solar heat, the drying will generally be done with warm air. So, solar systems can work with air or water system. The potential industries that can be benefited from such solar thermal energy systems are: bricks, plants, fruits, coffee, wood, textiles, leather, malt (kiln drying of green malt).

Akoy et al. described the design features, criteria and calculation of a solar dryer for a mango factory [65]. Fuller describes the theoretical and technical basics of solar drying systems, evaluated the performance and discussed future developments [28]. Mehrdadi et al. experimentally investigated the performance of a solar dryer used in industrial drying in pharmaceutical industries [51]. Rural Industries Research and Development Corporation worked on developing a solar drying machine for agricultural products [47]. Sebaii et al. reviewed the fundamentals and research work done in solar drying systems [18]. Lee studied and constructed solar drying system with an evacuated tube solar collector and also evaluated the system performance with indoor and outdoor drying [45]. Pirasteh et al. reviewed the development of solar drying applications in industry and agriculture, energy consumption capacity and aspects of using solar dryer from multiple viewpoints [70]. Hubackova et al. researches on solar dryers used in fish processing in Combodia [33]. Mustayen et al. studied and reviewed the performance studies on different solar dryers 
[61]. Yuan et al. researched on solar dryers used in carpet drying, thus evaluated its thermal performance [95]. Montero et al. researched on solar drying systems used in drying wastes from oliveoil mills [55]. Liu et al. reviewed 5 types of solar drying systems used in drying herbal Chinese medicine [46]. Kumar et al. reviewed and presented different types of solar dryers through a varied application areas and their progress [40].

Table 4 illustrates the main industrial application areas where a solar drying system is already in use, their key features, solar collector information and also operational temperature data.

\begin{tabular}{|c|c|c|c|c|c|}
\hline Industry & Country & Name & $\begin{array}{l}\text { Industrial } \\
\text { Operation }\end{array}$ & $\begin{array}{l}\text { Solar } \\
\text { Collecto } \\
r\end{array}$ & $\begin{array}{l}\text { Temperature }{ }^{\circ} \\
\text { C) }\end{array}$ \\
\hline \multirow[t]{2}{*}{$\begin{array}{l}\text { Brewerie } \\
\mathrm{s}\end{array}$} & $\begin{array}{l}\text { German } \\
y\end{array}$ & $\begin{array}{l}\text { Newmarket } \\
\text { er } \\
\text { Lammsbraw }\end{array}$ & $\begin{array}{l}\text { Drying } \\
\text { process }\end{array}$ & $\begin{array}{l}\text { Air } \\
\text { collector }\end{array}$ & $\sim 60$ \\
\hline & & $\begin{array}{l}\text { Carriers \& } \\
\text { Sons }\end{array}$ & Drying & $\begin{array}{l}\text { Air } \\
\text { collector }\end{array}$ & $\sim 43$ \\
\hline \multirow[t]{9}{*}{ Food } & \multirow[t]{4}{*}{ USA } & $\begin{array}{l}\text { Keyaqa } \\
\text { Orchards }\end{array}$ & $\begin{array}{l}\text { Walnuts } \\
\text { Drying }\end{array}$ & $\begin{array}{l}\text { Air } \\
\text { collector }\end{array}$ & $\sim 43$ \\
\hline & & $\begin{array}{l}\text { Kreher's } \\
\text { Poultry } \\
\text { Farms }\end{array}$ & $\begin{array}{l}\text { Drying of } \\
\text { chicken } \\
\text { Eggs }\end{array}$ & $\begin{array}{l}\text { Air } \\
\text { collector }\end{array}$ & \\
\hline & & $\begin{array}{l}\text { Stapleton } \\
\text { Spence } \\
\text { Fruit } \\
\text { Packing Co. }\end{array}$ & $\begin{array}{l}\text { Rehydrate } \\
\text { Dried } \\
\text { Foods }\end{array}$ & $\begin{array}{l}\text { Unglazed } \\
\text { Collector }\end{array}$ & \\
\hline & & $\begin{array}{l}\text { Sunsweet } \\
\text { Dryers }\end{array}$ & $\begin{array}{l}\text { Prune } \\
\text { Drying }\end{array}$ & $\begin{array}{l}\text { Air } \\
\text { collector }\end{array}$ & $10 \sim 15$ \\
\hline & India & $\begin{array}{l}\text { Kaveri } \\
\text { Agricare } \\
\text { Pvt. Ltd. }\end{array}$ & $\begin{array}{l}\text { Drying } \\
\text { Coir Peat }\end{array}$ & $\begin{array}{l}\text { Air } \\
\text { collector }\end{array}$ & $\sim 105$ \\
\hline & $\begin{array}{l}\text { Costa } \\
\text { Rica }\end{array}$ & Coopeldos & $\begin{array}{l}\text { Coffee } \\
\text { Drying }\end{array}$ & $\begin{array}{l}\text { Air } \\
\text { collector }\end{array}$ & $40 \sim 45$ \\
\hline & Panama & $\begin{array}{l}\text { Duren } \\
\text { Coffee }\end{array}$ & $\begin{array}{l}\text { Coffee } \\
\text { Drying }\end{array}$ & $\begin{array}{l}\text { Air } \\
\text { collector }\end{array}$ & $40 \sim 45$ \\
\hline & China & $\begin{array}{l}\text { Gengli Fruit } \\
\text { Drying }\end{array}$ & $\begin{array}{l}\text { Fruit } \\
\text { drying }\end{array}$ & $\begin{array}{l}\text { Air } \\
\text { collector }\end{array}$ & $50 \sim 70$ \\
\hline & $\begin{array}{l}\text { Indonesi } \\
\text { a }\end{array}$ & $\begin{array}{l}\text { Malabar } \\
\text { Tea Drying }\end{array}$ & Tea drying & $\begin{array}{l}\text { Air } \\
\text { collector }\end{array}$ & $\sim 35$ \\
\hline Mining & Austria & Korner Kvk & $\begin{array}{l}\text { Preheating } \\
\text {, drying }\end{array}$ & $\begin{array}{l}\text { Flat plate } \\
\text { collector }\end{array}$ & $50 \sim 80$ \\
\hline \multirow[t]{4}{*}{$\begin{array}{l}\text { Agricultur } \\
\text { e }\end{array}$} & India & $\begin{array}{l}\text { AMR dal } \\
\text { Mill }\end{array}$ & $\begin{array}{l}\text { Drying of } \\
\text { Pulse }\end{array}$ & $\begin{array}{l}\text { Air } \\
\text { collector }\end{array}$ & $65 \sim 75$ \\
\hline & $\begin{array}{l}\text { Romani } \\
\text { a }\end{array}$ & $\begin{array}{l}\text { Aroma } \\
\text { Plant } \\
\text { Romania }\end{array}$ & $\begin{array}{l}\text { Drying of } \\
\text { Medicinal } \\
\text { Plants }\end{array}$ & $\begin{array}{l}\text { Air } \\
\text { collector }\end{array}$ & \\
\hline & USA & $\begin{array}{l}\text { Sonoma } \\
\text { Country } \\
\text { Herb } \\
\text { Exchange }\end{array}$ & $\begin{array}{l}\text { Drying of } \\
\text { Herbs }\end{array}$ & $\begin{array}{l}\text { Air } \\
\text { collector }\end{array}$ & \\
\hline & $\begin{array}{l}\text { German } \\
y\end{array}$ & Krimmer & $\begin{array}{l}\text { Drying of } \\
\text { Wild } \\
\text { Flower } \\
\text { Seeds }\end{array}$ & $\begin{array}{l}\text { Flat plate } \\
\text { collector }\end{array}$ & \\
\hline Leather & India & Leo Leather & $\begin{array}{l}\text { Chemical } \\
\text { mixing, } \\
\text { drying }\end{array}$ & $\begin{array}{l}\text { Flat plate } \\
\text { collector }\end{array}$ & \\
\hline \multirow[t]{2}{*}{$\begin{array}{l}\text { Fabricate } \\
\text { d Metal }\end{array}$} & $\begin{array}{l}\text { German } \\
y\end{array}$ & $\begin{array}{l}\text { Lackiercent } \\
\text { er Shulte }\end{array}$ & $\begin{array}{l}\text { Drying } \\
\text { Chamber }\end{array}$ & $\begin{array}{l}\text { Evacuate } \\
\text { d tube } \\
\text { collector }\end{array}$ & \\
\hline & Portugal & $\begin{array}{l}\text { Silampos } \\
\text { S.A. }\end{array}$ & $\begin{array}{l}\text { Drying } \\
\text { finished } \\
\text { product }\end{array}$ & $\begin{array}{l}\text { Parallel } \\
\text { trough } \\
\text { collector }\end{array}$ & $50 \sim 160$ \\
\hline Tobacco & $\begin{array}{l}\text { Argentin } \\
\mathrm{a}\end{array}$ & $\begin{array}{l}\text { Grammer } \\
\text { Solar } \\
\text { Argentina }\end{array}$ & $\begin{array}{l}\text { Drying of } \\
\text { tobacco }\end{array}$ & $\begin{array}{l}\text { Air } \\
\text { collector }\end{array}$ & \\
\hline $\begin{array}{l}\text { Rubber \& } \\
\text { Plastic } \\
\text { Products }\end{array}$ & Thailand & $\begin{array}{l}\text { Inter } \\
\text { Rubber } \\
\text { Latex Co. } \\
\text { Ltd. }\end{array}$ & $\begin{array}{l}\text { Drying of } \\
\text { natural } \\
\text { rubber }\end{array}$ & $\begin{array}{l}\text { Air } \\
\text { collector }\end{array}$ & \\
\hline Concrete & Austria & $\begin{array}{l}\text { Leitt Beton } \\
\text { Gmbh }\end{array}$ & $\begin{array}{l}\text { Drying of } \\
\text { pre- } \\
\text { fabricated } \\
\text { concrete } \\
\text { component } \\
\mathrm{s}\end{array}$ & $\begin{array}{l}\text { Flat plate } \\
\text { collector }\end{array}$ & \\
\hline Furniture & Austria & $\begin{array}{l}\text { Carpenting } \\
\text { Hamminger }\end{array}$ & $\begin{array}{l}\text { Wood } \\
\text { Drying }\end{array}$ & $\begin{array}{l}\text { Flat plate } \\
\text { collector }\end{array}$ & $25 \sim 115$ \\
\hline
\end{tabular}

\begin{tabular}{|l|l|l|l|l|l|}
\hline $\begin{array}{l}\text { Repairing } \\
\&\end{array}$ & German & Lackiererei & $\begin{array}{l}\text { Drying } \\
\text { process }\end{array}$ & $\begin{array}{l}\text { Evacuate } \\
\text { d tube } \\
\text { collector }\end{array}$ & $22 \sim 24,60 \sim 70$ \\
$\mathrm{n}$ & $\mathrm{Vogel}$ & & & \\
\hline
\end{tabular}

Table 4: Industries working with solar drying process [101].
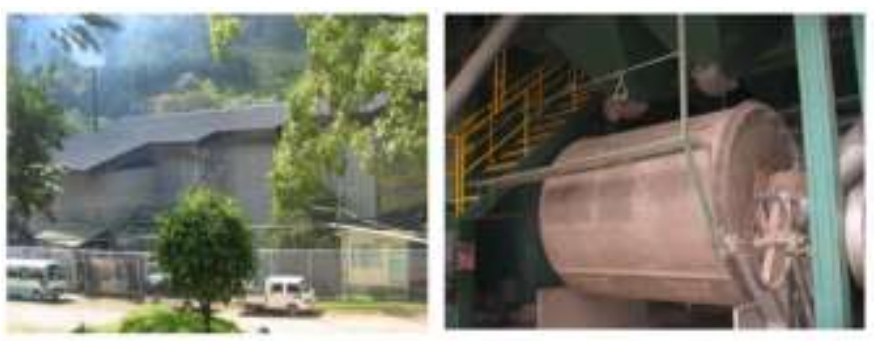

Fig.2. Copendos, Costa Rica coffee drying process [79].

\subsection{Preheating processes}

Solar thermal energy is ideal to use for preheating processes with lowtemperature applications. Waste heat, compressed air compressors, cooling equipment, gas engines might serve as alternative heat source. Typical examples can be: warm water and boiler feed water.

Celuppi et al. studied and analyzed the performance of 4 vacuum solar collectors for boiler water preheating [10]. Esen et al. investigated the performance of heat pump integrated solar space heating system $[19,20]$. Kabeel et al. reviewed the designs, and further improvement methods for solar air heater to reduce fuel consumption [37].

Table 5 illustrates the main industrial application areas where a solar preheating system is already in use, their key features, solar collector information and also operational temperature data.

\begin{tabular}{|c|c|c|c|c|c|}
\hline $\begin{array}{l}\text { Industr } \\
y\end{array}$ & Country & Name & $\begin{array}{l}\text { Industrial } \\
\text { Operation }\end{array}$ & $\begin{array}{l}\text { Solar } \\
\text { Collecto } \\
\mathbf{r}\end{array}$ & $\begin{array}{l}\text { Temperature }\left(^{\circ}\right. \\
\text { C) }\end{array}$ \\
\hline \multirow[t]{4}{*}{$\begin{array}{l}\text { Breweri } \\
\text { es }\end{array}$} & USA & Gatorade & $\begin{array}{l}\text { For soft } \\
\text { drink } \\
\text { production }\end{array}$ & $\begin{array}{l}\text { Flat plate } \\
\text { collector }\end{array}$ & $\sim 35$ \\
\hline & \multirow[t]{2}{*}{ Germany } & $\begin{array}{l}\text { Brauerei } \\
\text { Hald } \\
\text { (brewery } \\
\text { for } \\
\text { beverages) }\end{array}$ & $\begin{array}{l}\text { Preheating } \\
\text { of bottles }\end{array}$ & $\begin{array}{l}\text { Flat plate } \\
\text { collector }\end{array}$ & $40 \sim 120$ \\
\hline & & $\begin{array}{l}\text { Newmarket } \\
\text { er } \\
\text { Lammsbra } \\
\text { w }\end{array}$ & $\begin{array}{l}\text { For drying } \\
\text { process }\end{array}$ & $\begin{array}{l}\text { Air } \\
\text { collector }\end{array}$ & $\sim 60$ \\
\hline & $\begin{array}{l}\text { Czech } \\
\text { Republic }\end{array}$ & $\begin{array}{l}\text { Cider } \\
\text { House } \\
\text { Hostetin }\end{array}$ & $\begin{array}{l}\text { Cider } \\
\text { before } \\
\text { pasteurizati } \\
\text { on \& for } \\
\text { bottle } \\
\text { washing }\end{array}$ & $\begin{array}{l}\text { Flat plate } \\
\text { collector }\end{array}$ & \\
\hline \multirow[t]{5}{*}{ Food } & Mexico & $\begin{array}{l}\text { Durango } \\
\text { Dairy } \\
\text { Company }\end{array}$ & $\begin{array}{l}\text { Boiler } \\
\text { Preheating }\end{array}$ & $\begin{array}{l}\text { Parallel } \\
\text { trough } \\
\text { collector }\end{array}$ & $20 \sim 95$ \\
\hline & \multirow[t]{3}{*}{ Spain } & $\begin{array}{l}\text { Industria } \\
\text { Alimnetaria } \\
\text { Alcoyana }\end{array}$ & $\begin{array}{l}\text { Boiler feed } \\
\text { water for }\end{array}$ & $\begin{array}{l}\text { Flat plate } \\
\text { collector }\end{array}$ & $40 \sim 130$ \\
\hline & & $\begin{array}{l}\text { Metadero } \\
\text { Montesierr } \\
\text { a }\end{array}$ & $\begin{array}{l}\text { Boiler feed } \\
\text { water }\end{array}$ & $\begin{array}{l}\text { Flat plate } \\
\text { collector }\end{array}$ & $40 \sim 60$ \\
\hline & & TE-PE S.A. & $\begin{array}{l}\text { Boiler feed } \\
\text { water }\end{array}$ & $\begin{array}{l}\text { Flat plate } \\
\text { collector }\end{array}$ & $40 \sim 130$ \\
\hline & Netherlan & Perfetti van & Hot water & Flat plate & \\
\hline
\end{tabular}




\begin{tabular}{|l|l|l|l|l|l|}
\hline & ds & melle & $\begin{array}{l}\text { and process } \\
\text { heat }\end{array}$ & collector & \\
\cline { 2 - 6 } & China & $\begin{array}{l}\text { Gengli Fruit } \\
\text { Drying }\end{array}$ & $\begin{array}{l}\text { For fruit } \\
\text { drying }\end{array}$ & $\begin{array}{l}\text { Air } \\
\text { collector }\end{array}$ & $50 \sim 70$ \\
\hline Textiles & China & $\begin{array}{l}\text { Jiangsu } \\
\text { Printing \& } \\
\text { Dyeing }\end{array}$ & $\begin{array}{l}\text { For printing } \\
\text { \& dyeing } \\
\text { process }\end{array}$ & $\begin{array}{l}\text { Evacuat } \\
\text { ed tube } \\
\text { collector }\end{array}$ & $\sim 50$ \\
\hline Mining & Austria & Korner Kvk & $\begin{array}{l}\text { Preheating, } \\
\text { drying }\end{array}$ & $\begin{array}{l}\text { Flat plate } \\
\text { collector }\end{array}$ & $50 \sim 80$ \\
\hline
\end{tabular}

Table 5: Industries working with solar preheating process [101].

\subsection{Steam Heating Process}

Solar steam heating is done with conventional energy sources runs with smaller equipment like heat exchangers. To integrate a solar energy system, the design should be focused on operating at the lowest temperature levels after increasing collector efficiency. These applications constitute the major part of industrial solar thermal applications, with cost effectiveness of all the currently available solar thermal technologies.

Haagen et al. investigated the solar process steam systems installed in the pharmaceutical industry, RAM pharmaceuticals in Jordan [48]. Valenzuela et al. analyzed the numerical results obtained from the thermal-hydraulic behavior of solar fields producing steam using parabolic-trough collectors under different design conditions [93].

Table 6 illustrates the main industrial application areas where a solar steam heating system is already in use, their key features, solar collector information and also operational temperature data.

\begin{tabular}{|c|c|c|c|c|c|}
\hline Industry & Country & Name & $\begin{array}{l}\text { Industrial } \\
\text { Operatio } \\
\text { n }\end{array}$ & $\begin{array}{l}\text { Solar } \\
\text { Collecto } \\
r\end{array}$ & $\begin{array}{l}\text { Temperature }\left(^{\circ}\right. \\
\text { C) }\end{array}$ \\
\hline Food & USA & Frito Lay & $\begin{array}{l}\text { Steam for } \\
\text { Heating }\end{array}$ & $\begin{array}{l}\text { Parallel } \\
\text { trough } \\
\text { collector }\end{array}$ & $\sim 243$ \\
\hline & $\begin{array}{l}\text { Switzerlan } \\
\text { d }\end{array}$ & $\begin{array}{l}\text { Emmi } \\
\text { Dairy } \\
\text { Saignelsgi } \\
\text { er }\end{array}$ & $\begin{array}{l}\text { Different } \\
\text { drying } \\
\text { processe } \\
\mathrm{s}\end{array}$ & $\begin{array}{l}\text { Parallel } \\
\text { trough } \\
\text { collector }\end{array}$ & $140 \sim 180$ \\
\hline Textile & India & $\begin{array}{l}\text { Purple } \\
\text { Creations }\end{array}$ & $\begin{array}{l}\text { Steam } \\
\text { processin } \\
\text { g \& \& } \\
\text { washing }\end{array}$ & $\begin{array}{l}\text { Other or } \\
\text { various } \\
\text { collector } \\
\mathrm{s}\end{array}$ & \\
\hline Chemical & China & $\begin{array}{l}\text { Solar } \\
\text { steam } \\
\text { boiler for } \\
\text { procter \& } \\
\text { gamble } \\
\text { (Tianjin) }\end{array}$ & $\begin{array}{l}\text { Steam } \\
\text { generatio } \\
\mathrm{n}\end{array}$ & $\begin{array}{l}\text { Parallel } \\
\text { trough } \\
\text { collector }\end{array}$ & $\sim 130$ \\
\hline $\begin{array}{l}\text { Fabricate } \\
\text { d Metal }\end{array}$ & Germany & $\begin{array}{l}\text { Alanod } \\
\text { Solar }\end{array}$ & $\begin{array}{l}\text { Productio } \\
\text { n of } \\
\text { saturated } \\
\text { steam }\end{array}$ & $\begin{array}{l}\text { Parallel } \\
\text { trough } \\
\text { collector }\end{array}$ & $\sim 143$ \\
\hline
\end{tabular}

Table 6: Industries working with solar steam heating process [101].

\subsection{Pasteurization and sterilization process}

Dairy industries can utilize solar energy for process operations as they operate with no day off. Among the solar food processes, pasteurization and sterilization are the energy-intensive ones where major difference is the phase of the medium. Pasteurizing bottled requires a heat transfer medium because it is impossible to apply a direct heat exchange. The heat transfer medium can be connected to a solar thermal plant for pasteurizing and sterilization. It can be for liquid food, packaged food, devices, and other process streams.
Aiken et al. designed and developed a prototype solar water pasteurizer system with integral heat exchanger [2]. Paull submitted a report on solar pasteurization on water used for washing fruit and vegetable [68]. Rabab et al. fabricated and tested the performance of low temperature solar milk pasteurizer [73]. Osama et al. carried out a TRNSYS simulation to find a potential solar collector for sterilization applicationsm depending on industrial area [66].

Table 7 illustrates the main industrial application areas where a solar pasteurization or a solar sterilization system is already in use, their key features, solar collector information and also operational temperature data.

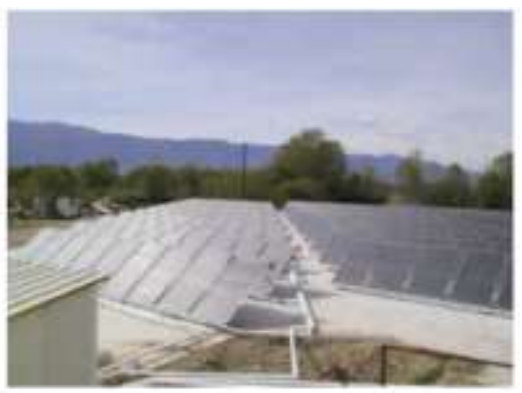

Fig. 3. $706 \mathrm{~kW}$ solar thermal plant in dairy industry in Greece [94].

\begin{tabular}{|c|c|c|c|c|c|}
\hline Industry & $\begin{array}{l}\text { Countr } \\
y\end{array}$ & Name & $\begin{array}{l}\text { Industrial } \\
\text { Operation }\end{array}$ & $\begin{array}{l}\text { Solar } \\
\text { Collecto } \\
\mathbf{r}\end{array}$ & $\begin{array}{l}\text { Temperature }\left(^{\circ}\right. \\
\text { C) }\end{array}$ \\
\hline \multirow[t]{3}{*}{$\begin{array}{l}\text { Brewerie } \\
\mathrm{s}\end{array}$} & Austria & $\begin{array}{l}\text { Gangl Fruit } \\
\text { Juices }\end{array}$ & $\begin{array}{l}\text { Pasteurizatio } \\
\mathrm{n}\end{array}$ & $\begin{array}{l}\text { Flat } \\
\text { plate } \\
\text { collector }\end{array}$ & $95 \sim 105$ \\
\hline & \multirow[t]{2}{*}{$\begin{array}{l}\text { Austria } \\
\text { Mexico }\end{array}$} & $\begin{array}{l}\text { Metbrauer } \\
\text { ei } \\
\text { Newwirth }\end{array}$ & $\begin{array}{l}\text { Pasteurizatio } \\
\mathrm{n} \text {, sterilization }\end{array}$ & $\begin{array}{l}\text { Flat } \\
\text { plate } \\
\text { collector }\end{array}$ & $50 \sim 95$ \\
\hline & & $\begin{array}{l}\text { Dairy Plant } \\
\text { Ladonita }\end{array}$ & $\begin{array}{l}\text { Pasteurizatio } \\
\mathrm{n}\end{array}$ & $\begin{array}{l}\text { Parallel } \\
\text { trough } \\
\text { collector }\end{array}$ & $60 \sim 95$ \\
\hline \multirow[t]{7}{*}{ Food } & \multirow[t]{3}{*}{$\begin{array}{l}\text { Mexico } \\
\text { India }\end{array}$} & $\begin{array}{l}\text { Nestle } \\
\text { Dairy Plant }\end{array}$ & $\begin{array}{l}\text { Pasteurizatio } \\
\mathrm{n}\end{array}$ & $\begin{array}{l}\text { Parallel } \\
\text { trough } \\
\text { collector }\end{array}$ & $80 \sim 95$ \\
\hline & & $\begin{array}{l}\text { Nestle } \\
\text { Dairy } \\
\text { Plant- } \\
\text { Lagos ds } \\
\text { Moreno } \\
\end{array}$ & $\begin{array}{l}\text { Pasteurizatio } \\
\mathrm{n}\end{array}$ & $\begin{array}{l}\text { Parallel } \\
\text { trough } \\
\text { collector }\end{array}$ & $80 \sim 95$ \\
\hline & & $\begin{array}{l}\text { B.G. } \\
\text { Chitale }\end{array}$ & $\begin{array}{l}\text { Pasteurizatio } \\
\mathrm{n} \text { of milks }\end{array}$ & $\begin{array}{l}\text { Other or } \\
\text { various } \\
\text { collector } \\
\mathrm{s}\end{array}$ & $\sim 150$ \\
\hline & \multirow[t]{4}{*}{$\begin{array}{l}\text { India } \\
\text { Austria }\end{array}$} & $\begin{array}{l}\text { Indian } \\
\text { Institute of } \\
\text { Horticultur } \\
\mathrm{e}\end{array}$ & $\begin{array}{l}\text { Pasteurizatio } \\
\mathrm{n} \text { of straw }\end{array}$ & $\begin{array}{l}\text { Other or } \\
\text { various } \\
\text { collector } \\
\mathrm{s}\end{array}$ & $80 \sim 100$ \\
\hline & & $\begin{array}{l}\text { Mahanand } \\
\text { a Dairy }\end{array}$ & $\begin{array}{l}\text { Pasteurizatio } \\
\mathrm{n} \text { of milks }\end{array}$ & $\begin{array}{l}\text { Other or } \\
\text { various } \\
\text { collector } \\
\text { s }\end{array}$ & $\sim 120$ \\
\hline & & $\begin{array}{l}\text { Milma } \\
\text { Dairy }\end{array}$ & $\begin{array}{l}\text { Pasteurizatio } \\
\mathrm{n} \text { of milks }\end{array}$ & $\begin{array}{l}\text { Flat } \\
\text { plate } \\
\text { collector }\end{array}$ & \\
\hline & & $\begin{array}{l}\text { Krispl Fruit } \\
\text { Juice }\end{array}$ & $\begin{array}{l}\text { Pasteur } \\
\text { crates } \\
\text { washing } \\
\text { space climate }\end{array}$ & $\begin{array}{l}\text { Flat } \\
\text { plate } \\
\text { collector }\end{array}$ & $\sim 80$ \\
\hline
\end{tabular}

Table 7: Industries working with solar pasteurization process [101]. 


\subsection{Washing processes}

Washing processes require a bulk of warm water, which provides an excellent opportunity to use solar thermal energy in practically all commercial sectors like bottles, barrels and containers in the food industry, metal parts and varnishing, galvanizing and enameling surfaces, and textile industry, business enterprises, laundries etc.

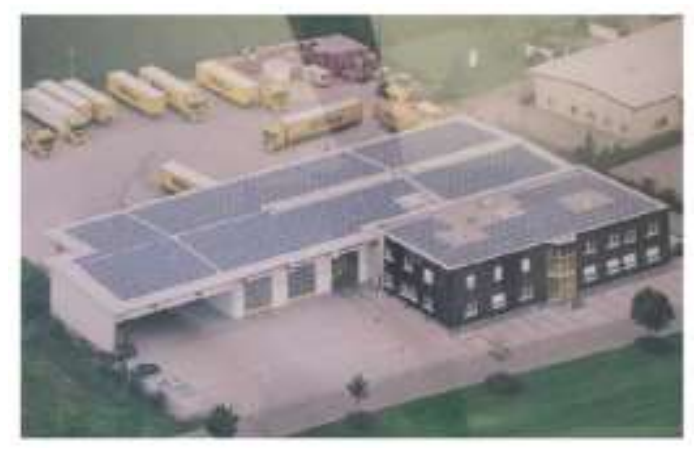

Fig. 4. Washing demonstration plant in Austria [95].

Table 8 illustrates the main industrial application areas where a solar washing system is already in use, their key features, solar collector information and also operational temperature data.

\begin{tabular}{|c|c|c|c|c|c|}
\hline Industry & $\begin{array}{l}\text { Countr } \\
y\end{array}$ & Name & $\begin{array}{l}\text { Industrial } \\
\text { Operation }\end{array}$ & $\begin{array}{l}\text { Solar } \\
\text { Collecto } \\
r\end{array}$ & $\begin{array}{l}\text { Temperature( } \\
\left.{ }^{\circ} \mathrm{C}\right)\end{array}$ \\
\hline $\begin{array}{l}\text { Automobi } \\
\text { le }\end{array}$ & India & $\begin{array}{l}\text { Mahindra } \\
\text { Vehicle } \\
\text { Manufacturer } \\
\text { s }\end{array}$ & $\begin{array}{l}\text { Engine } \\
\text { components }\end{array}$ & $\begin{array}{l}\text { Other or } \\
\text { various } \\
\text { collector } \\
\mathrm{s}\end{array}$ & $\sim 120$ \\
\hline \multirow[t]{11}{*}{$\begin{array}{l}\text { Brewerie } \\
\mathrm{s}\end{array}$} & Greece & $\begin{array}{l}\text { Achaia } \\
\text { Clauss S.A. }\end{array}$ & $\begin{array}{l}\text { Bottle } \\
\text { Washing }\end{array}$ & $\begin{array}{l}\text { Flat } \\
\text { plate } \\
\text { collector }\end{array}$ & $45 \sim 60$ \\
\hline & \multirow[t]{2}{*}{$\begin{array}{l}\text { Germa } \\
\text { ny }\end{array}$} & $\begin{array}{l}\text { Hofmuhl } \\
\text { Brewery }\end{array}$ & $\begin{array}{l}\text { Bottle } \\
\text { washing }\end{array}$ & $\begin{array}{l}\text { Evacuat } \\
\text { ed tube } \\
\text { collector }\end{array}$ & $20 \sim 110$ \\
\hline & & $\begin{array}{l}\text { Brauerei } \\
\text { Hald } \\
\text { (brewery for } \\
\text { beverages) }\end{array}$ & Cleaning & $\begin{array}{l}\text { Flat } \\
\text { plate } \\
\text { collector }\end{array}$ & $40 \sim 120$ \\
\hline & USA & $\begin{array}{l}\text { Brown's } \\
\text { Brewing Co. }\end{array}$ & Cleaning & $\begin{array}{l}\text { Flat } \\
\text { plate } \\
\text { collector }\end{array}$ & \\
\hline & France & Bourdoil & $\begin{array}{l}\text { Bottle } \\
\text { cleaning }\end{array}$ & $\begin{array}{l}\text { Flat } \\
\text { plate } \\
\text { collector }\end{array}$ & $15 \sim 70$ \\
\hline & $\begin{array}{l}\text { Czech } \\
\text { Republi } \\
\text { c }\end{array}$ & $\begin{array}{l}\text { Cider House } \\
\text { Hostetin }\end{array}$ & $\begin{array}{l}\text { Bottle } \\
\text { washing }\end{array}$ & $\begin{array}{l}\text { Flat } \\
\text { plate } \\
\text { collector }\end{array}$ & \\
\hline & $\begin{array}{l}\text { South } \\
\text { Africa }\end{array}$ & CBC Brewing & $\begin{array}{l}\text { Cleaning } \\
\text { process }\end{array}$ & $\begin{array}{l}\text { Flat } \\
\text { plate } \\
\text { collector }\end{array}$ & \\
\hline & \multirow[t]{3}{*}{ Austria } & $\begin{array}{l}\text { Metbrauerei } \\
\text { Newwirth }\end{array}$ & $\begin{array}{l}\text { Bottle } \\
\text { washing, }\end{array}$ & $\begin{array}{l}\text { Flat } \\
\text { plate } \\
\text { collector }\end{array}$ & $50 \sim 95$ \\
\hline & & $\begin{array}{l}\text { Petter } \\
\text { Vineyard }\end{array}$ & $\begin{array}{l}\text { Bottle } \\
\text { washing }\end{array}$ & $\begin{array}{l}\text { Flat } \\
\text { plate } \\
\text { collector }\end{array}$ & $20 \sim 90$ \\
\hline & & $\begin{array}{ll}\text { Gangl } & \text { Fruit } \\
\text { Juices } & \end{array}$ & $\begin{array}{l}\text { Bottle } \\
\text { cleaning, }\end{array}$ & $\begin{array}{l}\text { Flat } \\
\text { plate } \\
\text { collector }\end{array}$ & $95 \sim 105$ \\
\hline & $\begin{array}{l}\text { Saudi } \\
\text { Arabia }\end{array}$ & $\begin{array}{l}\text { Nestle } \\
\text { Waters }\end{array}$ & $\begin{array}{l}\text { Bottle } \\
\text { washing }\end{array}$ & $\begin{array}{l}\text { Flat } \\
\text { plate } \\
\text { collector }\end{array}$ & \\
\hline Food & Greece & Tyras S.A. & Washing of & Flat & $20 \sim 80$ \\
\hline
\end{tabular}

\begin{tabular}{|c|c|c|c|c|c|}
\hline & & & $\begin{array}{l}\text { cisterns and } \\
\text { lorries }\end{array}$ & $\begin{array}{l}\text { plate } \\
\text { collector }\end{array}$ & \\
\hline & & Mevgal S.A. & $\begin{array}{l}\text { Cleaning } \\
\text { equipment }\end{array}$ & $\begin{array}{l}\text { Other or } \\
\text { various } \\
\text { collector } \\
\mathrm{s}\end{array}$ & $20 \sim 90$ \\
\hline & & $\begin{array}{l}\text { Plektemboriki } \\
\text { Kritis S.A. }\end{array}$ & $\begin{array}{l}\text { Cleaning } \\
\text { Olives }\end{array}$ & $\begin{array}{l}\text { Flat } \\
\text { plate } \\
\text { collector }\end{array}$ & $40 \sim 90$ \\
\hline & \multirow[t]{3}{*}{ USA } & $\begin{array}{l}\text { Adams Farm } \\
\text { Slaughterhou } \\
\text { se }\end{array}$ & Cleaning & $\begin{array}{l}\text { Flat } \\
\text { plate } \\
\text { collector }\end{array}$ & \\
\hline & & $\begin{array}{l}\text { Battenhill } \\
\text { Valley } \\
\text { Creamery }\end{array}$ & Cleaning & $\begin{array}{l}\text { Flat } \\
\text { plate } \\
\text { collector }\end{array}$ & \\
\hline & & $\begin{array}{l}\text { Prestage } \\
\text { Foods }\end{array}$ & Cleaning & $\begin{array}{l}\text { Flat } \\
\text { plate } \\
\text { collector }\end{array}$ & $60 \sim 82$ \\
\hline & Austria & $\begin{array}{l}\text { Eidvogel } \\
\text { Hubert } \\
\text { Bernegger }\end{array}$ & $\begin{array}{l}\text { Fish } \\
\text { Transportati } \\
\text { on trucks }\end{array}$ & & \\
\hline & & $\begin{array}{l}\text { Krispl Fruit } \\
\text { Juice }\end{array}$ & $\begin{array}{l}\text { Washing } \\
\text { space }\end{array}$ & $\begin{array}{l}\text { Flat } \\
\text { plate } \\
\text { collector }\end{array}$ & $\sim 80$ \\
\hline & & $\begin{array}{l}\text { Moguntia } \\
\text { Spice Making }\end{array}$ & Cleaning & $\begin{array}{l}\text { Flat } \\
\text { plate } \\
\text { collector }\end{array}$ & \\
\hline & Israel & $\begin{array}{l}\text { Golan } \\
\text { Winery }\end{array}$ & $\begin{array}{l}\text { Barrel } \\
\text { Washing }\end{array}$ & $\begin{array}{l}\text { Flat } \\
\text { plate } \\
\text { collector }\end{array}$ & $\sim 95$ \\
\hline & India & $\begin{array}{l}\text { Kwality Walls } \\
\text { Inccream }\end{array}$ & Cleaning & $\begin{array}{l}\text { Flat } \\
\text { plate } \\
\text { collector }\end{array}$ & \\
\hline & $\begin{array}{l}\text { Portuga } \\
\text { I }\end{array}$ & $\begin{array}{l}\text { Knoww Best } \\
\text { Foods S.A. }\end{array}$ & $\begin{array}{l}\text { Tools } \\
\text { washing }\end{array}$ & $\begin{array}{l}\text { Flat } \\
\text { plate } \\
\text { collector }\end{array}$ & $40 \sim 45$ \\
\hline & Spain & $\begin{array}{l}\text { Montesano- } \\
\text { Jerez de los } \\
\text { caballero }\end{array}$ & $\begin{array}{l}\text { Raw } \\
\text { product } \\
\text { reception } \\
\text { washing }\end{array}$ & $\begin{array}{l}\text { Flat } \\
\text { plate } \\
\text { collector }\end{array}$ & $40 \sim 45$ \\
\hline & France & Bonilait Dairy & Cleaning & $\begin{array}{l}\text { Flat } \\
\text { plate } \\
\text { collector }\end{array}$ & $\sim 80$ \\
\hline & $\begin{array}{l}\text { Germa } \\
\text { ny }\end{array}$ & $\begin{array}{l}\text { Edmund- } \\
\text { gourmet } \\
\text { foods }\end{array}$ & $\begin{array}{l}\text { Rinsing \& } \\
\text { Cleaning of } \\
\text { bottle plants }\end{array}$ & $\begin{array}{l}\text { Flat } \\
\text { plate } \\
\text { collector }\end{array}$ & $20 \sim 60$ \\
\hline & Jordan & $\begin{array}{l}\text { Seniora Food } \\
\text { Industries }\end{array}$ & $\begin{array}{l}\text { Cleaning } \\
\text { requirement } \\
\mathrm{s}\end{array}$ & $\begin{array}{l}\text { Evacuat } \\
\text { ed tube } \\
\text { collector }\end{array}$ & \\
\hline \multirow[t]{3}{*}{ Textile } & Greece & $\begin{array}{l}\text { Allegro S.A. } \\
\text { Childrens' } \\
\text { clothing } \\
\text { manufacturer }\end{array}$ & $\begin{array}{l}\text { Washing } \\
\text { machines }\end{array}$ & $\begin{array}{l}\text { Flat } \\
\text { plate } \\
\text { collector }\end{array}$ & $33 \sim 60$ \\
\hline & \multirow[t]{2}{*}{ India } & $\begin{array}{l}\text { Purple } \\
\text { Creations }\end{array}$ & Washing & $\begin{array}{l}\text { Other or } \\
\text { various } \\
\text { collector } \\
\mathrm{s}\end{array}$ & \\
\hline & & $\begin{array}{l}\text { Sharman } \\
\text { Shawls }\end{array}$ & $\begin{array}{l}\text { Washing } \\
\text { garments }\end{array}$ & $\begin{array}{l}\text { Flat } \\
\text { plate } \\
\text { collector }\end{array}$ & $\sim 100$ \\
\hline $\begin{array}{l}\text { Wearing } \\
\text { Apparel }\end{array}$ & $\begin{array}{l}\text { Vietna } \\
\mathrm{m}\end{array}$ & Saitex Jeans & $\begin{array}{l}\text { Jeans } \\
\text { washing } \\
\text { process }\end{array}$ & $\begin{array}{l}\text { Flat } \\
\text { plate } \\
\text { collector }\end{array}$ & \\
\hline \multirow[t]{5}{*}{ Mining } & \multirow{5}{*}{$\begin{array}{l}\text { South } \\
\text { Africa }\end{array}$} & $\begin{array}{l}\text { Anglo Plant- } \\
\text { brakfontein }\end{array}$ & Cleaning & $\begin{array}{l}\text { Flat } \\
\text { plate } \\
\text { collector }\end{array}$ & \\
\hline & & $\begin{array}{l}\text { Anglo Plant- } \\
\text { middlepunt }\end{array}$ & Cleaning & $\begin{array}{l}\text { Flat } \\
\text { plate } \\
\text { collector }\end{array}$ & \\
\hline & & $\begin{array}{l}\text { BHP Bolliton } \\
\text { wolwekrans } \\
\text { colliery }\end{array}$ & Cleaning & $\begin{array}{l}\text { Flat } \\
\text { plate } \\
\text { collector }\end{array}$ & $\sim 60$ \\
\hline & & $\begin{array}{l}\text { Northern } \\
\text { Platinum's } \\
\text { Booysendal } \\
\text { Mine }\end{array}$ & Cleaning & $\begin{array}{l}\text { Flat } \\
\text { plate } \\
\text { collector }\end{array}$ & $\sim \sim 60$ \\
\hline & & $\begin{array}{l}\text { Xstrata } \\
\text { Elands Mine }\end{array}$ & Cleaning & $\begin{array}{l}\text { Evacuat } \\
\text { ed tube } \\
\text { collector }\end{array}$ & $\sim \sim 60$ \\
\hline Chemical & India & $\begin{array}{l}\text { L'oreal } \\
\text { Punne }\end{array}$ & $\begin{array}{l}\text { Cleaning } \\
\text { process }\end{array}$ & $\begin{array}{l}\text { Flat } \\
\text { plate }\end{array}$ & $\sim 55$ \\
\hline
\end{tabular}




\begin{tabular}{|l|l|l|l|l|l|}
\hline & & & & collector & \\
\cline { 2 - 6 } & Austria & MAPAG & $\begin{array}{l}\text { Cleaning of } \\
\text { samples }\end{array}$ & $\begin{array}{l}\text { Flat } \\
\text { plate } \\
\text { collector }\end{array}$ & $\sim 60$ \\
\hline $\begin{array}{l}\text { Fabricate } \\
\text { d Metal }\end{array}$ & France & $\begin{array}{l}\text { Viessmann } \\
\text { Faulquemont }\end{array}$ & $\begin{array}{l}\text { Cleaning } \\
\text { bath }\end{array}$ & $\begin{array}{l}\text { Evacuat } \\
\text { ed tube } \\
\text { collector }\end{array}$ & $\sim 60$ \\
\hline $\begin{array}{l}\text { Transport } \\
\text { Equipme } \\
\text { nt }\end{array}$ & India & $\begin{array}{l}\text { Harite } \\
\text { Seatings } \\
\text { Systems } \\
\text { Limited }\end{array}$ & $\begin{array}{l}\text { Cleaning } \\
\text { automobile } \\
\text { parts }\end{array}$ & $\begin{array}{l}\text { Evacuat } \\
\text { ed tube } \\
\text { collector }\end{array}$ & $55 \sim 60$ \\
\hline
\end{tabular}

Table 8: Industries working with solar washing and cleaning process [101].

10. Currently used Solar Industrial Process Heat SystemsSector-wise analysis

\subsection{Automobile Industry}

Automobile industries operate different types of production process which consume a vast amount of energy as electricity or heat. Energy sources used in automobile production processes are mainly electricity, petroleum products like natural gas and other sources.

Currently South Africa, India and Spain are employing solar process heating systems in their production systems. The operations are heating water used in the paint application process, washing the engine components in automobile manufacturing unit, bodyworks pretreatment line and bodyworks sheet surface preparation. Different types of solar collector are in use here and the maximum temperature that can be achieved is $120^{\circ} \mathrm{C}$. Typical production processes in the automobile industry which require heat energy explained below in detail, with the temperatures required in the process and the thermal energy transfer medium as hot water, hot air or pressured steam.

Zahler et al. investigated a solar-driven convection system installed in as automobile factory in Germany [96]. Uppal et al. reviewed solar industrial process heating systems in use in Indian automobile industries based on the most energy intensive process stages [90]. Table 9 depicts similar research on solar industrial process heating systems in use in automobile industries.

\begin{tabular}{|l|l|l|l|}
\hline Year & $\begin{array}{l}\text { Global } \\
\text { Analysis/Country } \\
\text { Specific }\end{array}$ & $\begin{array}{l}\text { Existing SHIP } \\
\text { Analysis }\end{array}$ & Reference \\
\hline 2012 & Germany & $\begin{array}{l}\text { Solar-driven } \\
\text { convection oven }\end{array}$ & {$[97]$} \\
\hline 2015 & India & $\begin{array}{l}\text { CST } \\
\text { technologies } \\
\text { based on } \\
\text { process stages }\end{array}$ & {$[90]$} \\
\hline
\end{tabular}

Table 9: Literature review for SIPH in automobile industries.

Table 10 presents the country-wise operational industries where solar process heat is in use and their manufacturing specifications.

\begin{tabular}{|l|l|l|l|l|}
\hline Country & Name & $\begin{array}{l}\text { Industrial } \\
\text { Operation }\end{array}$ & $\begin{array}{l}\text { Solar } \\
\text { Collector }\end{array}$ & Temperature $\left({ }^{\circ} \mathbf{C}\right)$ \\
\hline $\begin{array}{l}\text { South } \\
\text { Africa }\end{array}$ & $\begin{array}{l}\text { BMW } \\
\text { Manufacturing }\end{array}$ & $\begin{array}{l}\text { Hot water used } \\
\text { in the paint } \\
\text { application } \\
\text { process }\end{array}$ & $\begin{array}{l}\text { Evacuated } \\
\text { tube } \\
\text { collector }\end{array}$ & $\sim 90$ \\
\hline
\end{tabular}

\begin{tabular}{|l|l|l|l|l|}
\hline India & $\begin{array}{l}\text { Mahindra } \\
\text { Vehicle } \\
\text { Manufacturers }\end{array}$ & $\begin{array}{l}\text { Washing the } \\
\text { engine } \\
\text { components in } \\
\text { automobile } \\
\text { manufacturing } \\
\text { unit }\end{array}$ & $\begin{array}{l}\text { Other or } \\
\text { various } \\
\text { collectors }\end{array}$ & $\sim 120$ \\
\hline Spain & Nissan Avila & $\begin{array}{l}\text { Bodyworks pre- } \\
\text { treatment line }\end{array}$ & $\begin{array}{l}\text { Flat plate } \\
\text { collector }\end{array}$ & \\
\cline { 2 - 5 } & FASA & $\begin{array}{l}\text { Bodyworks plate } \\
\text { Sheet surface } \\
\text { preparation for } \\
\text { the welding } \\
\text { process \& } \\
\text { before the } \\
\text { painting }\end{array}$ & $\begin{array}{l}\text { Flat } \\
\text { collector }\end{array}$ & \\
& & & \\
\end{tabular}

Table 10: Existing SHIP in automobile industries [101].

\subsection{Breweries}

In the brewing industry, solar thermal can be used for processes such as: steam generation, malting process, stopping germination of grains, air cooling, conservation with hot air, power supplying of washing machines, withering and kiln processes. Malting plants and breweries consume heat for their thermally driven processes at temperature level ranges between $25^{\circ} \mathrm{C}$ and $120^{\circ} \mathrm{C}$. It seems feasible to supply heat at this temperature level which is not higher than the actual amount required but in reality it is often decided by the consumer needing the highest temperature. Several European countries, China, South Africa and USA are contributing for reduced carbon di-oxide emission by involving solar process heating in breweries. The respective process operations include: washing and cleaning, which is most widely used with maximum temperatures of $120^{\circ} \mathrm{C}$. Then comes the preheating of bottles done with air collector or flat-plate collector which delivers maximum $60^{\circ} \mathrm{C}$ temperature. There could be several operations like cooling of wine cellar $\left(95^{\circ} \mathrm{C}\right)$, pasteurization and sterilization. These processes are categorized briefly on Table 12.

Lauterbach et al. reviewed solar process heating system integration problems and also their potential in breweries [43]. Mauthner et al. suggested solution to the integration problems of solar industrial process heating systems working from flat-plate solar collectors in brewing $[49,50]$.

Table 11 presents the country-wise operational industries where solar process heat is in use and their manufacturing specifications.

\begin{tabular}{|c|c|c|c|c|}
\hline Country & Name & $\begin{array}{l}\text { Industrial } \\
\text { Operation }\end{array}$ & $\begin{array}{l}\text { Solar } \\
\text { Collector }\end{array}$ & Temperature $\left({ }^{\circ} \mathrm{C}\right)$ \\
\hline Greece & $\begin{array}{l}\text { Achaia } \\
\text { Clauss S.A. }\end{array}$ & $\begin{array}{l}\text { Bottle washing } \\
\text { in winery }\end{array}$ & $\begin{array}{l}\text { Flat plate } \\
\text { collector }\end{array}$ & $45 \sim 60$ \\
\hline \multirow[t]{4}{*}{ USA } & $\begin{array}{l}\text { Barrington } \\
\text { Brewery \& } \\
\text { Restaurant }\end{array}$ & $\begin{array}{l}\text { Brewing } \\
\text { process }\end{array}$ & $\begin{array}{l}\text { Flat plate } \\
\text { collector }\end{array}$ & \\
\hline & $\begin{array}{l}\text { Brown's } \\
\text { Brewing Co. }\end{array}$ & Cleaning & $\begin{array}{l}\text { Flat plate } \\
\text { collector }\end{array}$ & \\
\hline & Gatorade & $\begin{array}{l}\text { Preheating the } \\
\text { water for soft } \\
\text { drink production }\end{array}$ & $\begin{array}{l}\text { Flat plate } \\
\text { collector }\end{array}$ & $\sim 35$ \\
\hline & $\begin{array}{l}\text { Milwaukee } \\
\text { Brewing Co. }\end{array}$ & $\begin{array}{l}\text { Hot water } \\
\text { consumption }\end{array}$ & $\begin{array}{l}\text { Flat plate } \\
\text { collector }\end{array}$ & \\
\hline \multirow[t]{2}{*}{ France } & Bourdoil & $\begin{array}{l}\text { Bottle cleaning } \\
\text { with tap water }\end{array}$ & $\begin{array}{l}\text { Flat plate } \\
\text { collector }\end{array}$ & $15 \sim 70$ \\
\hline & $\begin{array}{l}\text { GICB Wine } \\
\text { Cellers }\end{array}$ & $\begin{array}{l}\text { Cooling of wine } \\
\text { celler }\end{array}$ & $\begin{array}{l}\text { Evacuated } \\
\text { tube } \\
\text { collector }\end{array}$ & $70 \sim 95$ \\
\hline Germany & $\begin{array}{l}\text { Brauerei } \\
\text { Hald }\end{array}$ & $\begin{array}{l}\text { Cleaning \& } \\
\text { thermal }\end{array}$ & $\begin{array}{l}\text { Flat plate } \\
\text { collector }\end{array}$ & $40 \sim 120$ \\
\hline
\end{tabular}




\begin{tabular}{|c|c|c|c|c|}
\hline & $\begin{array}{l}\text { (brewery for } \\
\text { beverages) }\end{array}$ & $\begin{array}{l}\text { preheating of } \\
\text { bottles }\end{array}$ & & \\
\hline & $\begin{array}{l}\text { Hofmuhl } \\
\text { Brewery }\end{array}$ & $\begin{array}{l}\text { Brewing water, } \\
\text { bottle washing, } \\
\text { defrosting \& } \\
\text { space heating }\end{array}$ & $\begin{array}{l}\text { Evacuated } \\
\text { tube } \\
\text { collector }\end{array}$ & $20 \sim 110$ \\
\hline & Hutt Brewery & $\begin{array}{l}\text { Heating the } \\
\text { brewing water }\end{array}$ & $\begin{array}{l}\text { Flat plate } \\
\text { collector }\end{array}$ & $40 \sim 95$ \\
\hline & $\begin{array}{l}\text { Newmarketer } \\
\text { Lammsbraw }\end{array}$ & $\begin{array}{l}\text { Preheating the } \\
\text { ambient air for } \\
\text { drying process }\end{array}$ & $\begin{array}{l}\text { Air } \\
\text { collector }\end{array}$ & $\sim 60$ \\
\hline $\begin{array}{l}\text { South } \\
\text { Africa }\end{array}$ & $\begin{array}{l}\text { CBC } \\
\text { Brewing }\end{array}$ & $\begin{array}{l}\text { Brewing water } \\
\& \quad \text { cleaning } \\
\text { process }\end{array}$ & $\begin{array}{l}\text { Flat plate } \\
\text { collector }\end{array}$ & \\
\hline \multirow[t]{2}{*}{ China } & $\begin{array}{l}\text { Chongqing } \\
\text { Beverage }\end{array}$ & $\begin{array}{l}\text { Heating for } \\
\text { warehouse in } \\
\text { winter, hot } \\
\text { water supply for } \\
\text { production in } \\
\text { other seasons }\end{array}$ & $\begin{array}{l}\text { Evacuated } \\
\text { tube } \\
\text { collector }\end{array}$ & \\
\hline & $\begin{array}{l}\text { Coca cola } \\
\text { Shanghai }\end{array}$ & $\begin{array}{l}\text { Other process } \\
\text { heating }\end{array}$ & $\begin{array}{l}\text { Evacuated } \\
\text { tube } \\
\text { collector }\end{array}$ & \\
\hline $\begin{array}{l}\text { Czech } \\
\text { Republic }\end{array}$ & $\begin{array}{l}\text { Cider House } \\
\text { Hostetin }\end{array}$ & $\begin{array}{l}\text { Preheating of } \\
\text { cider before } \\
\text { pasteurization \& } \\
\text { for bottle } \\
\text { washing }\end{array}$ & $\begin{array}{l}\text { Flat plate } \\
\text { collector }\end{array}$ & \\
\hline \multirow{4}{*}{ Austria } & $\begin{array}{ll}\text { Gangl Fruit } \\
\text { Juices }\end{array}$ & $\begin{array}{l}\text { Bottle cleaning, } \\
\text { pasteurization }\end{array}$ & $\begin{array}{l}\text { Flat plate } \\
\text { collector }\end{array}$ & $95 \sim 105$ \\
\hline & $\begin{array}{l}\text { Goess } \\
\text { Brewer } \\
\text { Mashing } \\
\text { Process } \\
\end{array}$ & & $\begin{array}{l}\text { Flat plate } \\
\text { collector }\end{array}$ & $80 \sim 90$ \\
\hline & $\begin{array}{l}\text { Metbrauerei } \\
\text { Newwirth }\end{array}$ & $\begin{array}{l}\text { Bottle washing, } \\
\text { pasteurization, } \\
\text { sterilization }\end{array}$ & $\begin{array}{l}\text { Flat plate } \\
\text { collector }\end{array}$ & $50 \sim 95$ \\
\hline & $\begin{array}{l}\text { Petter } \\
\text { Vineyard } \\
\end{array}$ & $\begin{array}{l}\text { Wine cooling, } \\
\text { bottle washing }\end{array}$ & $\begin{array}{l}\text { Flat plate } \\
\text { collector }\end{array}$ & $20 \sim 90$ \\
\hline $\begin{array}{l}\text { Saudi } \\
\text { Arabia }\end{array}$ & $\begin{array}{l}\text { Nestle } \\
\text { Waters }\end{array}$ & $\begin{array}{l}\text { Hot water for } \\
\text { bottle washing }\end{array}$ & $\begin{array}{l}\text { Flat plate } \\
\text { collector }\end{array}$ & \\
\hline Tunisia & $\begin{array}{l}\text { Winery } \\
\text { Grombalia }\end{array}$ & $\begin{array}{l}\text { Solar cooling by } \\
\text { feeding an } \\
\text { ammonia-water } \\
\text { by absorption } \\
\text { chiller }\end{array}$ & $\begin{array}{l}\text { Fresnel } \\
\text { collector }\end{array}$ & $\sim 110, \sim 160$ \\
\hline
\end{tabular}

Table 11: Existing SHIP in breweries [101].

\begin{tabular}{|l|l|l|l|}
\hline Year & $\begin{array}{l}\text { Global } \\
\text { Analysis/Country } \\
\text { Specific }\end{array}$ & $\begin{array}{l}\text { Existing SHIP } \\
\text { Analysis }\end{array}$ & Reference \\
\hline 2009 & Germany & $\begin{array}{l}\text { SHIP systems } \\
\text { in Hutt Brewery } \\
\text { were analyzed }\end{array}$ & {$[43]$} \\
\hline 2014 & Global & $\begin{array}{l}\text { 3 large scale } \\
\text { solar thermal } \\
\text { systems are } \\
\text { analyzed }\end{array}$ & {$[49,50]$} \\
\hline
\end{tabular}

Table 12: Literature review for SIPH in brewing.

To utilize the organic matter from brewing residues, optimized heat recovery can supply its thermal energy demand over resources. Theoretically, the full energy potential is not utilized so the pathway is the conventional means of energy. However, the conversion of heat from energy gained from renewable energy resources can ensure environment friendly beer production process for future. To increase the overall energy efficiency, the integration of solar heat energy gained from solar thermal collector technologies can be considered as a prospective alternative to fulfill the demands of typical low temperature process heat.

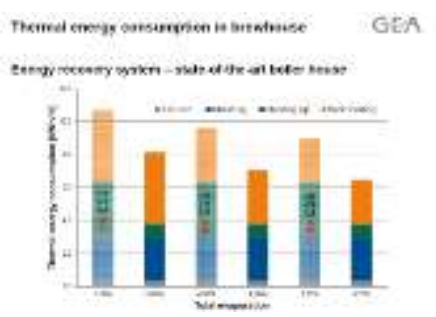

Fig. 5. Thermal processes and associated process temperatures in malting plants and breweries $[49,50]$.

\subsection{Food Industry}

The most common industrial processes where industrial process heat in the medium temperature range is in use are: pasteurizing, sterilizing, hydrolyzing, drying, evaporation, distillation, cleaning and washing; each of which is in the scope of food industry. The food industry is identified as the dominant industrial sector now a day for solar process heating systems. Most of the countries in Europe, North America, South America and Asia use industrial process heating systems in their own industries. The dominant ones are Mexico, USA, Greece, India, Spain, and Austria. The common process operations in food industries are water heating, pasteurization, cleaning, preheating, drying and cooking. Flat-plate solar collectors and parallel-trough collectors are generally in use here. Currently food industries are dealing with maximum $243^{\circ} \mathrm{C}$ where most of them are low-temperature process heat applications summarized in Table 14 below. Table 13 lists existing research and review describing solar industrial process heating systems in food industries.

\begin{tabular}{|l|l|l|l|}
\hline Year & $\begin{array}{l}\text { Global } \\
\text { Analysis/Country } \\
\text { Specific }\end{array}$ & Existing SHIP Analysis & Reference \\
\hline 2009 & India & $\begin{array}{l}\text { Indian technologies and } \\
\text { applications were analyzed }\end{array}$ & {$[67]$} \\
\hline 2011 & Global & $\begin{array}{l}\text { Integration feasibility analysis by } \\
\text { mathematical modelling }\end{array}$ & {$[72]$} \\
\hline 2012 & Global & $\begin{array}{l}\text { Different devices in use with } \\
\text { solar energy }\end{array}$ & {$[24]$} \\
\hline 2013 & Global & Discussed the potential of SHIP & {$[27]$} \\
\hline 2013 & Global & Different devices are analyzed & {$[14]$} \\
\hline 2013 & Global & $\begin{array}{l}\text { Small and medium scale } \\
\text { industry thermal energy } \\
\text { requirements }\end{array}$ & {$[63]$} \\
\hline
\end{tabular}

Table 13: Literature review for SIPH in food industries.

Kalogirou gave an overview on the solar industrial process heat potential in the food industry through solar collector analysis, annual energy gain and economic analysis [39]. Palaniappan described the roof mounted solar hot air technology adopted in various food processing systems in India [67]. Desai et al. discussed different devices in use with solar energy in the dairy industries [14]. Ramos et al. studied parabolic trough solar collector based solar process heat applications in Mexico [74]. Eswara et al. reviewed the solar food 
processing technologies based on solar collectors, concentrators and dryers in food processing [24]. Sharma et al. investigated the potential of solar industrial process heating integration in Indian dairy industry through solar radiation analysis [80]. Quijera et al. evaluated the feasibility of integrating solar thermal system into dairy industries by mathematical modeling [72].

Table 14 presents the country-wise operational industries where solar process heat is in use and their manufacturing specifications.

\begin{tabular}{|c|c|c|c|c|}
\hline Country & Name & $\begin{array}{l}\text { Industrial } \\
\text { Operation }\end{array}$ & $\begin{array}{l}\text { Solar } \\
\text { Collector }\end{array}$ & $\begin{array}{l}\text { Temperature } \\
\left({ }^{\circ} \mathrm{C}\right)\end{array}$ \\
\hline \multirow{8}{*}{ Mexico } & $\begin{array}{l}\text { Aviary Food } \\
\text { Pellet El Paisa }\end{array}$ & Heating water & $\begin{array}{l}\text { Parallel } \\
\text { trough } \\
\text { collector }\end{array}$ & $\sim 95$ \\
\hline & $\begin{array}{l}\text { Dairy } \\
\text { Indio }\end{array}$ & Process heat & $\begin{array}{l}\text { Parallel } \\
\text { trough } \\
\text { collector }\end{array}$ & $20 \sim 95$ \\
\hline & $\begin{array}{ll}\text { Dairy } & \text { Plant } \\
\text { Ladonita } & \end{array}$ & Pasteurization & $\begin{array}{l}\text { Parallel } \\
\text { trough } \\
\text { collector }\end{array}$ & $60 \sim 95$ \\
\hline & $\begin{array}{l}\text { Durango Dairy } \\
\text { Company }\end{array}$ & $\begin{array}{l}\text { Boiler } \\
\text { preheating }\end{array}$ & $\begin{array}{l}\text { Parallel } \\
\text { trough } \\
\text { collector }\end{array}$ & $20 \sim 95$ \\
\hline & $\begin{array}{ll}\text { Food } & \text { Pellet } \\
\text { Plant } & \end{array}$ & Cooking & $\begin{array}{l}\text { Parallel } \\
\text { trough } \\
\text { collector }\end{array}$ & $40 \sim 95$ \\
\hline & Matalan Dairy & $\begin{array}{l}\text { General } \\
\text { process } \\
\text { heating }\end{array}$ & $\begin{array}{l}\text { Parallel } \\
\text { trough } \\
\text { collector }\end{array}$ & $80 \sim 100$ \\
\hline & $\begin{array}{ll}\text { Nestle } & \text { Dairy } \\
\text { Plant } & \end{array}$ & Pasteurization & $\begin{array}{l}\text { Parallel } \\
\text { trough } \\
\text { collector }\end{array}$ & $80 \sim 95$ \\
\hline & $\begin{array}{l}\text { Nestle Dairy } \\
\text { Plant-Lagos ds } \\
\text { Moreno }\end{array}$ & Pasteurization & $\begin{array}{l}\text { Parallel } \\
\text { trough } \\
\text { collector }\end{array}$ & $80 \sim 95$ \\
\hline \multirow{9}{*}{ USA } & $\begin{array}{l}\text { Adams Farm } \\
\text { Slaughterhouse }\end{array}$ & Cleaning & $\begin{array}{l}\text { Flat plate } \\
\text { collector }\end{array}$ & \\
\hline & $\begin{array}{l}\text { Battenhill } \\
\text { Valley } \\
\text { Creamery }\end{array}$ & Cleaning & $\begin{array}{l}\text { Flat plate } \\
\text { collector }\end{array}$ & \\
\hline & $\begin{array}{ll}\text { Carriers } \quad \& \\
\text { Sons }\end{array}$ & Drying & $\begin{array}{l}\text { Air } \\
\text { collector }\end{array}$ & $\sim 43$ \\
\hline & Frito Lay & $\begin{array}{ll}\text { Steam for } \\
\text { heating }\end{array}$ & $\begin{array}{l}\text { Parallel } \\
\text { trough } \\
\text { collector }\end{array}$ & $\sim 243$ \\
\hline & $\begin{array}{l}\text { Keyaqa } \\
\text { Orchards }\end{array}$ & $\begin{array}{l}\text { Walnuts } \\
\text { drying }\end{array}$ & $\begin{array}{l}\text { Air } \\
\text { collector }\end{array}$ & $\sim 43$ \\
\hline & $\begin{array}{l}\text { Kreher's } \\
\text { Poultry Farms }\end{array}$ & $\begin{array}{l}\text { Drying of } \\
\text { chicken Eggs }\end{array}$ & $\begin{array}{l}\text { Air } \\
\text { collector }\end{array}$ & \\
\hline & $\begin{array}{l}\text { Prestage } \\
\text { Foods }\end{array}$ & Cleaning & $\begin{array}{l}\text { Flat plate } \\
\text { collector }\end{array}$ & $60 \sim 82$ \\
\hline & $\begin{array}{l}\text { Stapleton } \\
\text { Spence Fruit } \\
\text { Packing Co. }\end{array}$ & $\begin{array}{l}\text { Rehydrate } \\
\text { dried foods }\end{array}$ & $\begin{array}{l}\text { Unglazed } \\
\text { Collector }\end{array}$ & \\
\hline & $\begin{array}{l}\text { Sunsweet } \\
\text { Dryers }\end{array}$ & Prune drying & $\begin{array}{l}\text { Air } \\
\text { collector }\end{array}$ & $10 \sim 15$ \\
\hline \multirow{5}{*}{ Greece } & Alpino S.A. & $\begin{array}{l}\text { Hot water } \\
\text { preheating in } \\
\text { steam boiler }\end{array}$ & $\begin{array}{l}\text { Flat plate } \\
\text { collector }\end{array}$ & $20 \sim 70$ \\
\hline & $\begin{array}{l}\text { Mandrekas } \\
\text { S.A. }\end{array}$ & $\begin{array}{l}\text { Preservation } \\
\text { of yogurt } \\
\text { making } \\
\text { temperature }\end{array}$ & $\begin{array}{l}\text { Flat plate } \\
\text { collector }\end{array}$ & $40 \sim 45$ \\
\hline & Mevgal S.A. & $\begin{array}{l}\text { Hot water for } \\
\text { cleaning } \\
\text { equipment }\end{array}$ & $\begin{array}{l}\text { Other or } \\
\text { various } \\
\text { collectors }\end{array}$ & $20 \sim 90$ \\
\hline & $\begin{array}{l}\text { Plektemboriki } \\
\text { Kritis S.A. }\end{array}$ & $\begin{array}{l}\text { Cleaning } \\
\text { olives }\end{array}$ & $\begin{array}{l}\text { Flat plate } \\
\text { collector }\end{array}$ & $40 \sim 90$ \\
\hline & Tyras S.A. & $\begin{array}{l}\text { Washing of } \\
\text { cisterns and } \\
\text { lorries }\end{array}$ & $\begin{array}{l}\text { Flat plate } \\
\text { collector }\end{array}$ & $20 \sim 80$ \\
\hline \multirow{4}{*}{ India } & B.G. Chitale & $\begin{array}{l}\text { Pasteurization } \\
\text { of milks }\end{array}$ & $\begin{array}{l}\text { Other or } \\
\text { various } \\
\text { collectors }\end{array}$ & $\sim 150$ \\
\hline & $\begin{array}{l}\text { Indian Institute } \\
\text { of Horticulture }\end{array}$ & $\begin{array}{l}\text { Pasteurization } \\
\text { of straw }\end{array}$ & $\begin{array}{l}\text { Other or } \\
\text { various } \\
\text { collectors }\end{array}$ & $80 \sim 100$ \\
\hline & $\begin{array}{l}\text { Kaveri Agricare } \\
\text { Pvt. Ltd. }\end{array}$ & $\begin{array}{l}\text { Drying coir } \\
\text { Peat }\end{array}$ & $\begin{array}{l}\text { Air } \\
\text { collector }\end{array}$ & $\sim 105$ \\
\hline & Kwality Walls & Cleaning and & Flat plate & \\
\hline
\end{tabular}

\begin{tabular}{|c|c|c|c|c|}
\hline & Inccream & $\begin{array}{l}\text { making sugar } \\
\text { solution }\end{array}$ & collector & \\
\hline & $\begin{array}{l}\text { Mahananda } \\
\text { Dairy }\end{array}$ & $\begin{array}{l}\text { Pasteurization } \\
\text { of milks }\end{array}$ & $\begin{array}{l}\text { Other or } \\
\text { various } \\
\text { collectors }\end{array}$ & $\sim 120$ \\
\hline & Milma Dairy & $\begin{array}{l}\text { Pasteurization } \\
\text { of milks }\end{array}$ & $\begin{array}{l}\text { Flat plate } \\
\text { collector }\end{array}$ & \\
\hline & $\begin{array}{l}\text { Panchmahal } \\
\text { Dairy }\end{array}$ & $\begin{array}{l}\text { Preheat boiler } \\
\text { feed }\end{array}$ & $\begin{array}{l}\text { Flat plate } \\
\text { collector }\end{array}$ & \\
\hline & Tapi Foods & $\begin{array}{l}\text { Preheat boiler } \\
\text { feed }\end{array}$ & $\begin{array}{l}\text { Other or } \\
\text { various } \\
\text { collectors }\end{array}$ & \\
\hline France & Bonilait Dairy & Cleaning & $\begin{array}{l}\text { Flat plate } \\
\text { collector }\end{array}$ & $\sim 80$ \\
\hline South Africa & $\begin{array}{l}\text { Chalmar } \quad \text { Beef } \\
\text { Pty(Ltd) }\end{array}$ & Ablution & $\begin{array}{l}\text { Flat plate } \\
\text { collector }\end{array}$ & \\
\hline Costa Rica & Coopeldos & Coffee drying & $\begin{array}{l}\text { Air } \\
\text { collector }\end{array}$ & $40 \sim 45$ \\
\hline \multirow[t]{3}{*}{ Switzerland } & Cremo S.A. & $\begin{array}{l}\text { Milk } \\
\text { processing, } \\
\text { Coffee creme } \\
\text { Production }\end{array}$ & $\begin{array}{l}\text { Parallel } \\
\text { trough } \\
\text { collector }\end{array}$ & $\sim 150, \sim 110$ \\
\hline & $\begin{array}{l}\text { Emmi Dairy } \\
\text { Saignelsgier }\end{array}$ & $\begin{array}{ll}\text { Steam for } \\
\text { different } \\
\text { drying } \\
\text { processes }\end{array}$ & $\begin{array}{l}\text { Parallel } \\
\text { trough } \\
\text { collector }\end{array}$ & $140 \sim 180$ \\
\hline & Lesa Dairy & $\begin{array}{l}\text { Milk } \\
\text { processing }\end{array}$ & $\begin{array}{l}\text { Parallel } \\
\text { trough } \\
\text { collector }\end{array}$ & \\
\hline Panama & Duren Coffee & Coffee drying & $\begin{array}{l}\text { Air } \\
\text { collector }\end{array}$ & $40 \sim 45$ \\
\hline Germany & $\begin{array}{l}\text { Edmund- } \\
\text { gourmet foods }\end{array}$ & $\begin{array}{l}\text { Rinsing \& } \\
\text { cleaning of } \\
\text { bottle plants }\end{array}$ & $\begin{array}{l}\text { Flat plate } \\
\text { collector }\end{array}$ & $20 \sim 60$ \\
\hline \multirow{5}{*}{ Austria } & $\begin{array}{l}\text { Eidvogel } \\
\text { Hubert } \\
\text { Bernegger }\end{array}$ & $\begin{array}{l}\text { Washing of } \\
\text { fish } \\
\text { transportation } \\
\text { trucks }\end{array}$ & & \\
\hline & $\begin{array}{l}\text { Fleischwaren } \\
\text { berger }\end{array}$ & Cooking & $\begin{array}{l}\text { Flat plate } \\
\text { collector }\end{array}$ & $30 \sim 95$ \\
\hline & $\begin{array}{l}\text { Futtermittel } \\
\text { Fruit Juice }\end{array}$ & $\begin{array}{l}\text { Heating of } \\
\text { makeup water }\end{array}$ & $\begin{array}{l}\text { Flat plate } \\
\text { collector }\end{array}$ & \\
\hline & $\begin{array}{l}\text { Krispl Fruit } \\
\text { Juice }\end{array}$ & $\begin{array}{l}\text { Fruit pasteur } \\
\text { crates } \\
\text { washing } \\
\text { space climate }\end{array}$ & $\begin{array}{l}\text { Flat plate } \\
\text { collector }\end{array}$ & $\sim 80$ \\
\hline & $\begin{array}{l}\text { Moguntia Spice } \\
\text { Making }\end{array}$ & $\begin{array}{l}\text { Hot water for } \\
\text { cleaning and } \\
\text { production } \\
\text { process }\end{array}$ & $\begin{array}{l}\text { Flat plate } \\
\text { collector }\end{array}$ & \\
\hline \multirow[t]{2}{*}{ China } & $\begin{array}{ll}\text { Gengli } & \text { Fruit } \\
\text { Drying } & \end{array}$ & $\begin{array}{l}\text { Heating air for } \\
\text { fruit drying }\end{array}$ & $\begin{array}{l}\text { Air } \\
\text { collector }\end{array}$ & $50 \sim 70$ \\
\hline & $\begin{array}{l}\text { Meihao Food } \\
\text { Processing }\end{array}$ & $\begin{array}{l}\text { Sterilization } \\
\text { by hot water }\end{array}$ & $\begin{array}{l}\text { Evacuated } \\
\text { tube } \\
\text { collector }\end{array}$ & $\sim 80$ \\
\hline Israel & Golan Winery & $\begin{array}{l}\text { Barrel } \\
\text { washing }\end{array}$ & $\begin{array}{l}\text { Flat plate } \\
\text { collector }\end{array}$ & $2 \sim 95$ \\
\hline Portugal & $\begin{array}{l}\text { Knoww Best } \\
\text { Foods S.A. }\end{array}$ & $\begin{array}{l}\text { Hot water for } \\
\text { tools washing }\end{array}$ & $\begin{array}{l}\text { Flat plate } \\
\text { collector }\end{array}$ & $40 \sim 45$ \\
\hline Indonesia & $\begin{array}{ll}\text { Malabar } & \text { Tea } \\
\text { Drying } & \\
\end{array}$ & Tea drying & $\begin{array}{l}\text { Air } \\
\text { collector }\end{array}$ & $\sim 35$ \\
\hline \multirow{5}{*}{ Spain } & $\begin{array}{l}\text { Industria } \\
\text { Alimnetaria } \\
\text { Alcoyana }\end{array}$ & $\begin{array}{l}\text { Preheating of } \\
\text { boiler feed } \\
\text { water for olive } \\
\text { preparation }\end{array}$ & $\begin{array}{l}\text { Flat plate } \\
\text { collector }\end{array}$ & $40 \sim 130$ \\
\hline & $\begin{array}{l}\text { Metadero } \\
\text { Montesierra }\end{array}$ & $\begin{array}{l}\text { Preheating of } \\
\text { boiler feed } \\
\text { water }\end{array}$ & $\begin{array}{l}\text { Flat plate } \\
\text { collector }\end{array}$ & $40 \sim 60$ \\
\hline & $\begin{array}{l}\text { Montesano- } \\
\text { canary islands }\end{array}$ & $\begin{array}{l}\text { Hot water } \\
\text { used for wash } \\
\text { meat } \\
\text { derivatives }\end{array}$ & $\begin{array}{l}\text { Flat plate } \\
\text { collector }\end{array}$ & $40 \sim 60$ \\
\hline & $\begin{array}{l}\text { Montesano- } \\
\text { Jerez de los } \\
\text { caballero }\end{array}$ & $\begin{array}{l}\text { Raw product } \\
\text { reception } \\
\text { washing }\end{array}$ & $\begin{array}{l}\text { Flat plate } \\
\text { collector }\end{array}$ & $40 \sim 45$ \\
\hline & TE-PE S.A. & $\begin{array}{l}\text { Preheating of } \\
\text { boiler feed } \\
\text { water }\end{array}$ & $\begin{array}{l}\text { Flat plate } \\
\text { collector }\end{array}$ & $40 \sim 130$ \\
\hline Italy & $\begin{array}{l}\text { Nuova Sarda } \\
\text { Industria } \\
\text { Caaseria }\end{array}$ & $\begin{array}{l}\text { Other process } \\
\text { heating }\end{array}$ & $\begin{array}{l}\text { Fresnel } \\
\text { collector }\end{array}$ & $\sim 200$ \\
\hline Czech & PETA Bohemia & Hot water for & Flat plate & $10 \sim 90$ \\
\hline
\end{tabular}




\begin{tabular}{|l|l|l|l|l|}
\hline Republic & $\begin{array}{l}\text { Pekarna } \\
\text { Sobeslav }\end{array}$ & bakery & collector & \\
\hline Netherlands & $\begin{array}{l}\text { Perfetti van } \\
\text { melle }\end{array}$ & $\begin{array}{l}\text { Preheating of } \\
\text { hot water and } \\
\text { process heat }\end{array}$ & $\begin{array}{l}\text { Flat plate } \\
\text { collector }\end{array}$ & \\
\hline Jordan & $\begin{array}{l}\text { Seniora Food } \\
\text { Industries }\end{array}$ & $\begin{array}{l}\text { Hot water for } \\
\text { degreasing \& } \\
\text { cleaning } \\
\text { requirements }\end{array}$ & $\begin{array}{l}\text { Evacuated } \\
\text { tube } \\
\text { collector }\end{array}$ & \\
\hline
\end{tabular}

Table 14: Existing SHIP in food industries [101].

\subsection{Paper Industry}

The Indian paper industry accounts for about $2.6 \%$ of the total production of paper in the world. Fuel consumption in the paper industry in India comprises electricity, biomass, coal and petroleum fuel. To reduce greenhouse gas effects, fossil fuel consumption needs to be reduced besides maintaining the required energy supply to the paper industry, which can only be achieved by improving the fuel utilization efficiency and substituting the fossil fuel by harnessing renewable sources of energy. $75 \%$ of the total energy used in paper industry is essential for process heating at low and intermediate temperatures. Processes like bleaching and washing with hot water, pulping, drying, and boiler feed water heating consume most of the process heat, using heat transfer medium like water, steam, air or oil depending on specific process requirements. The paper industries still lack proper attention to reduce greenhouse gas emissions through proper utilization of renewable energy resources. Only in India B.S. paper mill is using solar heat for different process operations using various collectors integrated into the system with a maximum achieved temperature of $98^{\circ} \mathrm{C}$ (Table 16). There are a few literatures on SIPH in the paper industries (table 15).

\begin{tabular}{|l|l|l|l|}
\hline Year & $\begin{array}{l}\text { Global } \\
\text { Analysis/Country } \\
\text { Specific }\end{array}$ & $\begin{array}{l}\text { Existing SHIP } \\
\text { Analysis }\end{array}$ & Reference \\
\hline 2015 & India & $\begin{array}{l}\text { Potential } \\
\text { estimation of } \\
\text { SHIP }\end{array}$ & {$[79]$} \\
\hline 2016 & India & $\begin{array}{l}\text { potential } \\
\text { estimation of } \\
\text { SHIP and } \\
\text { corresponding } \\
\text { carbon-di-oxide } \\
\text { emissions } \\
\text { mitigation }\end{array}$ & {$[81]$} \\
& & \\
\hline
\end{tabular}

Table 15: Literature review for SIPH in paper industries.

Sharma et al. analyzed solar industrial process heating potential in paper industry in several research works. They had a preliminary assessment to estimate the SHIP potential by classifying paper mills, estimating the annual process heating requirements, analyzing solar radiation availability $[79,81]$.

\begin{tabular}{|l|l|l|l|l|}
\hline Country & Name & $\begin{array}{l}\text { Industrial } \\
\text { Operation }\end{array}$ & $\begin{array}{l}\text { Solar } \\
\text { Collector }\end{array}$ & Temperature $\left({ }^{\circ} \mathbf{C}\right)$ \\
\hline India & $\begin{array}{l}\text { B.S. } \\
\text { Paper Mill }\end{array}$ & $\begin{array}{l}\text { Different } \\
\text { process } \\
\text { applications }\end{array}$ & $\begin{array}{l}\text { Other or } \\
\text { various } \\
\text { collectors }\end{array}$ & $90 \sim 98$ \\
\hline
\end{tabular}

Table 16: Existing SHIP in paper industries [101].

\subsection{Pharmaceutical Industry}

The pharmaceutical industry has a substantial energy demand for producing pharmaceutical products, and for the formulation of the final product. The process temperature ranges around $160^{\circ} \mathrm{C}-180^{\circ} \mathrm{C}$ and depends on the process and the specific product. In Jordan this pharmaceutical industry contributes for around $20 \%$ to the manufacturing GDP. Pharmaceutical industries are identified as most promising industrial sector for European and North American countries where economy is dependent upon these kinds of industries. Now a days only Egypt and Greece are dealing with solar process heating based pharmaceutical industries for process steam generation and cooling, where several other operations operate at low temperature (Table 18). Table 17 lists the research on solar industrial process heating systems in pharmaceutical industries.

\begin{tabular}{|l|l|l|l|}
\hline Year & $\begin{array}{l}\text { Global } \\
\text { Analysis/Country } \\
\text { Specific }\end{array}$ & $\begin{array}{l}\text { Existing SHIP } \\
\text { Analysis }\end{array}$ & Reference \\
\hline 2014 & Jordan & $\begin{array}{l}\text { Solar process } \\
\text { steam for RAM } \\
\text { pharmacy }\end{array}$ & {$[48]$} \\
\hline
\end{tabular}

Table 17: Literature review for SIPH in pharmaceutical industries.

Haagen et al. estimated the solar process heating potential in the steam generation process in pharmaceutical industries [48].

\begin{tabular}{|l|l|l|l|l|}
\hline Country & Name & $\begin{array}{l}\text { Industrial } \\
\text { Operation }\end{array}$ & $\begin{array}{l}\text { Solar } \\
\text { Collector }\end{array}$ & Temperature $\left({ }^{\circ} \mathbf{C}\right)$ \\
\hline Egypt & $\begin{array}{l}\text { El Nasr } \\
\text { Pharmaceutical }\end{array}$ & $\begin{array}{l}\text { Production of } \\
\text { process } \\
\text { steam }\end{array}$ & $\begin{array}{l}\text { Parallel } \\
\text { trough } \\
\text { collector }\end{array}$ & $\sim 173$ \\
\hline Greece & Sarantis S.A. & $\begin{array}{l}\text { Warehouse } \\
\text { cooling }\end{array}$ & $\begin{array}{l}\text { Flat plate } \\
\text { collector }\end{array}$ & $7 \sim 45$ \\
\hline
\end{tabular}

Table 18. Existing SHIP in pharmaceutical industries [101].

\subsection{Textile Industry}

The textile industry requires a continuous supply of water like most of other industrial sectors, mainly for the dying process. The temperature requirement for the water is not only at a normal level but also at a higher level like $80^{\circ} \mathrm{C}$, which requires a considerable amount of heat energy. If conventional energy sources are used consequently for water heating, environmental impacts will also increase as a consequence. Solar water heating using solar process heat is a potential candidate to replace conventional fossil fuel sources in the textile industry to substantially reduce the environmental impact. Among the seven countries where textile industries are equipped with solar process heating systems, Greece, China and India are dominant ones. Water heating is the common process operation working with flat-plate collectors to obtain a maximum $100^{\circ} \mathrm{C}$ temperature. Other process operations include solar preheating, painting and textile production processes which are summarized in Table 20. Table 19 describes the literature on textile industry based process heating systems. 


\begin{tabular}{|l|l|l|l|}
\hline Year & $\begin{array}{l}\text { Global } \\
\text { Analysis/Country } \\
\text { Specific }\end{array}$ & $\begin{array}{l}\text { Existing SHIP } \\
\text { Analysis }\end{array}$ & Reference \\
\hline 2006 & Pakistan & $\begin{array}{l}\text { Alternating } \\
\text { solution to solar } \\
\text { water heating }\end{array}$ & {$[59]$} \\
\hline 2008 & Turkey & $\begin{array}{l}\text { Experimental } \\
\text { investigation of } \\
\text { solar water } \\
\text { heating }\end{array}$ & {$[58]$} \\
\hline 2013 & Global & $\begin{array}{l}\text { Small and } \\
\text { medium scale } \\
\text { industry }\end{array}$ & {$[74]$} \\
\hline 2015 & Global & $\begin{array}{l}\text { A medium scale } \\
\text { industry }\end{array}$ & {$[30]$} \\
\hline
\end{tabular}

Table 19: Literature review for SIPH in textile industries.

Muneer et al. investigated the thermal performance of two differently constructed solar water heaters to find out the potential in textile industries [58, 59]. Ramos et al. studied parabolic-trough solar collector based solar process heat applications in Mexico [74]. Frey et al. monitored the results of the solar industrial process heating systems installed in the textile industry of Germany based on real-time operational data [30].

\begin{tabular}{|c|c|c|c|c|}
\hline Country & Name & $\begin{array}{l}\text { Industrial } \\
\text { Operation }\end{array}$ & $\begin{array}{l}\text { Solar } \\
\text { Collector }\end{array}$ & Temperature $\left({ }^{\circ} \mathrm{C}\right)$ \\
\hline USA & Acme McCrary & $\begin{array}{l}\text { Hot water for } \\
\text { textile drying } \\
\text { process }\end{array}$ & $\begin{array}{l}\text { Flat plate } \\
\text { collector }\end{array}$ & \\
\hline \multirow[t]{2}{*}{ Greece } & $\begin{array}{l}\text { Allegro S.A. } \\
\text { Childrens' } \\
\text { clothing } \\
\text { manufacturer }\end{array}$ & $\begin{array}{l}\text { Hot water for } \\
\text { washing } \\
\text { machines }\end{array}$ & $\begin{array}{l}\text { Flat plate } \\
\text { collector }\end{array}$ & $33 \sim 60$ \\
\hline & $\begin{array}{l}\text { Kastrinagiannis } \\
\text { S.A. }\end{array}$ & $\begin{array}{l}\text { Hot water } \\
\text { directly for } \\
\text { dyeing } \\
\text { machines }\end{array}$ & $\begin{array}{l}\text { Flat plate } \\
\text { collector }\end{array}$ & $40 \sim 90$ \\
\hline \multirow[t]{2}{*}{ China } & Daly Textile & $\begin{array}{l}\text { Water } \\
\text { heating for } \\
\text { dyeing } \\
\text { process }\end{array}$ & $\begin{array}{l}\text { Flat plate } \\
\text { collector }\end{array}$ & $\sim 55$ \\
\hline & $\begin{array}{l}\text { Jiangsu Printing } \\
\text { \& Dyeing }\end{array}$ & $\begin{array}{l}\text { Solar } \\
\text { preheating } \\
\text { for printing \& } \\
\text { dyeing } \\
\text { process }\end{array}$ & $\begin{array}{l}\text { Evacuated } \\
\text { tube } \\
\text { collector }\end{array}$ & $\sim 50$ \\
\hline Vietnam & $\begin{array}{l}\text { Grammer Solar } \\
\text { Vietnam }\end{array}$ & $\begin{array}{l}\text { Textile } \\
\text { production } \\
\text { process }\end{array}$ & $\begin{array}{l}\text { Air } \\
\text { collector }\end{array}$ & \\
\hline Spain & Harlequin & Painting & $\begin{array}{l}\text { Flat plate } \\
\text { collector }\end{array}$ & \\
\hline Germany & Meiser Textile & $\begin{array}{l}\text { Other } \\
\text { process } \\
\text { heating }\end{array}$ & $\begin{array}{l}\text { Parallel } \\
\text { trough } \\
\text { collector }\end{array}$ & $\sim 140$ \\
\hline \multirow[t]{2}{*}{ India } & $\begin{array}{l}\text { Purple } \\
\text { Creations }\end{array}$ & $\begin{array}{l}\text { Iron the } \\
\text { children's } \\
\text { garments- } \\
\text { steam } \\
\text { processing \& } \\
\text { washing }\end{array}$ & $\begin{array}{l}\text { Other or } \\
\text { various } \\
\text { collectors }\end{array}$ & \\
\hline & $\begin{array}{l}\text { Sharman } \\
\text { Shawls }\end{array}$ & $\begin{array}{l}\text { Hot water } \\
\text { requirements } \\
\text { for dyeing, } \\
\text { bleaching \& } \\
\text { washing } \\
\text { garments }\end{array}$ & $\begin{array}{l}\text { Flat plate } \\
\text { collector }\end{array}$ & $\sim 100$ \\
\hline
\end{tabular}

Table 20: Existing SHIP in textile industries [101].

\subsection{Minerals processing}

Solar process heating technologies can be used for moderate temperature heating and steam generation, which are useful processes in the minerals processing industries. These SHIP applications would be particularly convenient in remote mines where fuel costs are much more significant than in urban areas. Lowtemperatures gas or liquid heating can be achieved with solar thermal systems. Solar heating systems with fluid temperatures range up to $150^{\circ} \mathrm{C}$ can be easily achieved with non-concentrating technologies while temperatures up to $400^{\circ} \mathrm{C}$ can be achieved using simple CST technologies. Potential applications of CST in minerals processing can be divided into process group- thermal processes, chemical processes, and carbothermic reduction processes. Solar thermal processes include solar kiln development for thermal decomposition of limestone $(\mathrm{CaCO})$ to produce lime $(\mathrm{CaO})$ at up to $1130^{\circ} \mathrm{C}$. Solar thermal heating of air for industrial applications has also been proposed, for example a material with high thermal conductivity and heat capacity might be used to form a heated permeable bed using solar thermal energy. Heated air then passes through the bed where temperatures of up to $850^{\circ} \mathrm{C}$ can be achieved. Preheated air can be used for metallurgical smelting processes like regenerative heating. Cowper stoves, the best-known high-temperature application, produce air heating for blast furnaces for iron production. SolarGas (CSIRO, New South Wales, and Australia) is natural gas reformed using CST energy to produce carbon monoxide and hydrogen gas (syngas) etc. CSIRO has developed the process to meet thermal energy requirement from solar energy. South Africa, Chile, Austria and Germany are dominant and vibrant mining based industrialized countries where solar process heating system is already built in and operated in mines, mostly for cleaning, mining, water heating operations. Other mine specific operations involve- copper electro-winning process, nickel bath, degreasing chemicals with flat-plate collector and evaporated-tube collector (Table 22). The mining sector still lacks where there are huge scopes for low-temperature, medium-temperature and also hightemperature solar process heating systems to get integrated for reducing greenhouse gas emissions. According to the mining industries, there are very few solar process heating systems (Table 21).

\begin{tabular}{|l|l|l|l|}
\hline Year & $\begin{array}{l}\text { Global } \\
\text { Analysis/Country } \\
\text { Specific }\end{array}$ & $\begin{array}{l}\text { Existing SHIP } \\
\text { Analysis }\end{array}$ & Reference \\
\hline 1999 & Global & $\begin{array}{l}\text { Replacement of } \\
\text { Hall-Heroult } \\
\text { process by solar } \\
\text { process heat }\end{array}$ & {$[60]$} \\
\hline 2013 & Australia & $\begin{array}{l}\text { Potential } \\
\text { processes } \\
\text { identification by } \\
\text { temperature } \\
\text { range }\end{array}$ & {$[15]$} \\
\hline 2015 & $\begin{array}{l}\text { Australia/ } \\
\text { Northern } \\
\text { Territory }\end{array}$ & $\begin{array}{l}\text { Considering } \\
\text { Northern } \\
\text { Territory's } \\
\text { potential for } \\
\text { solar power }\end{array}$ & {$[5]$} \\
\hline
\end{tabular}

Table 21: Literature review for SIPH in mining industries.

Dinter et al. studied the potential of solar industrial process heat on the ferroalloy industry, depending on energy requirements, sizing of collector fields and financial evaluation [15]. Murray proposed an intermediate $\mathrm{A} 1 \mathrm{~N}$ compound to replace the Hall-Heroult process 
through solar process heat [60]. Eglinton et al. studied concentrated solar thermal integration's potential in medium and high temperature process stages in mineral industries [11]. Baig et al. investigated the potential of concentrated solar power in remote mine sites in the Northern Territory of Australia [5].

\begin{tabular}{|c|c|c|c|c|}
\hline Country & Name & $\begin{array}{l}\text { Industrial } \\
\text { Operation }\end{array}$ & $\begin{array}{l}\text { Solar } \\
\text { Collector }\end{array}$ & Temperature $\left({ }^{\circ} \mathrm{C}\right)$ \\
\hline \multirow{5}{*}{$\begin{array}{l}\text { South } \\
\text { Africa }\end{array}$} & $\begin{array}{l}\text { Anglo Plant- } \\
\text { brakfontein }\end{array}$ & Cleaning & $\begin{array}{l}\text { Flat plate } \\
\text { collector }\end{array}$ & \\
\hline & $\begin{array}{l}\text { Anglo Plant- } \\
\text { middlepunt }\end{array}$ & Cleaning & $\begin{array}{l}\text { Flat plate } \\
\text { collector }\end{array}$ & \\
\hline & $\begin{array}{l}\text { BHP Bolliton } \\
\text { wolwekrans } \\
\text { colliery }\end{array}$ & $\begin{array}{l}\text { Mining \& } \\
\text { Cleaning }\end{array}$ & $\begin{array}{l}\text { Flat plate } \\
\text { collector }\end{array}$ & $\sim 60$ \\
\hline & $\begin{array}{l}\text { Northern } \\
\text { Platinum's } \\
\text { Booysendal } \\
\text { Mine }\end{array}$ & $\begin{array}{l}\text { Mining \& } \\
\text { cleaning }\end{array}$ & $\begin{array}{l}\text { Flat plate } \\
\text { collector }\end{array}$ & $\sim 60$ \\
\hline & $\begin{array}{l}\text { Xstrata } \\
\text { Elands Mine }\end{array}$ & $\begin{array}{l}\text { Mining } \quad \& \\
\text { cleaning }\end{array}$ & $\begin{array}{l}\text { Evacuated } \\
\text { tube } \\
\text { collector }\end{array}$ & $\sim 60$ \\
\hline \multirow[t]{2}{*}{ Chile } & $\begin{array}{l}\text { Codelco } \\
\text { Gabriela } \\
\text { Mistral }\end{array}$ & $\begin{array}{l}\text { Electro } \\
\text { winning } \\
\text { Process }\end{array}$ & $\begin{array}{l}\text { Flat plate } \\
\text { collector }\end{array}$ & $\sim 50$ \\
\hline & $\begin{array}{l}\text { Minera } \\
\text { Constanza }\end{array}$ & $\begin{array}{l}\text { Electro } \\
\text { winning } \\
\text { Process }\end{array}$ & $\begin{array}{l}\text { Flat plate } \\
\text { collector }\end{array}$ & \\
\hline Cyprus & $\begin{array}{l}\text { Hellenic } \\
\text { Copper Mine }\end{array}$ & $\begin{array}{l}\text { Industrial } \\
\text { mining, } \\
\text { leaching, } \\
\text { extraction }\end{array}$ & $\begin{array}{l}\text { Flat plate } \\
\text { collector }\end{array}$ & \\
\hline \multirow[t]{2}{*}{ Austria } & $\begin{array}{l}\text { Gillich } \\
\text { Galavanic }\end{array}$ & $\begin{array}{l}\text { Degreasing \& } \\
\text { removal of } \\
\text { lacquer from } \\
\text { metal parts in } \\
\text { baths }\end{array}$ & $\begin{array}{l}\text { Evacuated } \\
\text { tube } \\
\text { collector }\end{array}$ & $\sim 75$ \\
\hline & Korner Kvk & $\begin{array}{l}\text { Preheating, } \\
\text { drying }\end{array}$ & $\begin{array}{l}\text { Flat plate } \\
\text { collector }\end{array}$ & $50 \sim 80$ \\
\hline India & $\begin{array}{l}\text { Kangaroo } \\
\text { India Limited }\end{array}$ & Nickel bath & $\begin{array}{l}\text { Flat plate } \\
\text { collector }\end{array}$ & \\
\hline \multirow[t]{3}{*}{ Germany } & $\begin{array}{l}\text { Hustert } \\
\text { Galvanic }\end{array}$ & $\begin{array}{l}\text { Heating } \\
\text { thermal baths } \\
\text { for } \\
\text { electroplating }\end{array}$ & $\begin{array}{l}\text { Evacuated } \\
\text { tube } \\
\text { collector }\end{array}$ & $\sim 80$ \\
\hline & $\begin{array}{l}\text { Schiffer } \\
\text { Gmbh \& Co } \\
\text { KG }\end{array}$ & $\begin{array}{l}\text { Hot water for } \\
\text { galvanic baths }\end{array}$ & $\begin{array}{l}\text { Evacuated } \\
\text { tube } \\
\text { collector }\end{array}$ & $40 \sim 70$ \\
\hline & $\begin{array}{l}\text { Steinbach } \\
\text { und } \\
\text { Vallmann }\end{array}$ & $\begin{array}{l}\text { Hot water for } \\
\text { galvanic baths }\end{array}$ & $\begin{array}{l}\text { Evacuated } \\
\text { tube } \\
\text { collector }\end{array}$ & $60 \sim 80$ \\
\hline
\end{tabular}

Table 22: Existing SHIP in mineral industries [101].

\subsection{Manufacturing of Chemical Products}

There are various chemical production processes that require heat at a relatively low temperature level where primary preheating steps with other energy sources are supplementarily used by solar energy. There are areas within solar industrial process heating in the chemical industry that work exclusively at a low-process temperature. The most common processes where solar water heating is in use are water heating, steam heating, cleaning and painting process with flat-plate collector where currently the maximum attained temperature is $130^{\circ} \mathrm{C}$ (Table 24).

\begin{tabular}{|l|l|l|l|l|}
\hline Country & Name & $\begin{array}{l}\text { Industrial } \\
\text { Operation }\end{array}$ & $\begin{array}{l}\text { Solar } \\
\text { Collector }\end{array}$ & Temperature $\left({ }^{\circ} \mathbf{C}\right)$ \\
\hline India & L'oreal Punne & $\begin{array}{l}\text { Hot water for } \\
\text { cleaning process }\end{array}$ & $\begin{array}{l}\text { Flat plate } \\
\text { collector }\end{array}$ & $\sim 55$ \\
\hline Austria & MAPAG & $\begin{array}{l}\text { Cleaning of } \\
\text { samples for } \\
\text { analytical lab }\end{array}$ & $\begin{array}{l}\text { Flat plate } \\
\text { collector }\end{array}$ & $\sim 60$ \\
\hline Egypt & $\begin{array}{l}\text { Pachin Paints } \\
\text { Co. }\end{array}$ & $\begin{array}{l}\text { Heating of } \\
\text { chemicals }\end{array}$ & $\begin{array}{l}\text { Flat plate } \\
\text { collector }\end{array}$ & $\sim 50$ \\
\hline
\end{tabular}

\begin{tabular}{|l|l|l|l|l|}
\hline Germany & $\begin{array}{l}\text { Penzkofer } \\
\text { Autolackiererei }\end{array}$ & Painting process & $\begin{array}{l}\text { Air } \\
\text { collector }\end{array}$ & \\
\hline China & $\begin{array}{l}\text { Solar steam } \\
\text { boiler for } \\
\text { procter \& } \\
\text { gamble } \\
\text { (Tianjin) }\end{array}$ & Steam & $\begin{array}{l}\text { Parallel } \\
\text { trough } \\
\text { collector }\end{array}$ & $\sim 130$ \\
& & & \\
\hline
\end{tabular}

Table 23: Existing SHIP in chemical industries [101].

Meier et al. developed solar chemical reactor technology for reducing carbon di-oxide emissions in the lime and cement industry. Table 23 presents the country-wise operational industries where solar process heat is in use and their manufacturing specifications [52].

\begin{tabular}{|l|l|l|l|}
\hline Year & $\begin{array}{l}\text { Global } \\
\text { Analysis/Country } \\
\text { Specific }\end{array}$ & $\begin{array}{l}\text { Existing SHIP } \\
\text { Analysis }\end{array}$ & Reference \\
\hline 2014 & Spain & $\begin{array}{l}\text { Process heat } \\
\text { driven } \\
\text { vegetable } \\
\text { preservation }\end{array}$ & {$[85]$} \\
\hline 2015 & Global & $\begin{array}{l}\text { Solar process } \\
\text { heating systems } \\
\text { in agricultural } \\
\text { industries }\end{array}$ & {$[34]$} \\
\hline
\end{tabular}

Table 24: Literature review for SIPH in agricultural industries.

10.9. Agricultural Industry:

Solar industrial process heat is used extensively in the agricultural and fisheries industries from different countries, mainly for drying of agricultural products, water heating using different types of solar collectors which vary based on plant capacity and temperature-range requirements. Most of the industries have low-pressure temperature range applications with air collector, flat-plate collector, parallel troughcollector, etc. (Table 25). Table 24 describes the literature on such agricultural industries.

\begin{tabular}{|l|l|l|l|l|}
\hline Country & Name & $\begin{array}{l}\text { Industrial } \\
\text { Operation }\end{array}$ & $\begin{array}{l}\text { Solar } \\
\text { Collector }\end{array}$ & Temperature $\left(^{\circ} \mathbf{C}\right)$ \\
\hline India & $\begin{array}{l}\text { AMR dal } \\
\text { Mill }\end{array}$ & $\begin{array}{l}\text { Drying of } \\
\text { pulse }\end{array}$ & $\begin{array}{l}\text { Air } \\
\text { collector }\end{array}$ & $65 \sim 75$ \\
\hline Spain & $\begin{array}{l}\text { Acuinova } \\
\text { Andalucia } \\
\text { S.A. }\end{array}$ & $\begin{array}{l}\text { Heating } \\
\text { water }\end{array}$ & $\begin{array}{l}\text { Flat plate } \\
\text { collector }\end{array}$ & $23 \sim 26$ \\
\hline Romania & $\begin{array}{l}\text { Aroma Plant } \\
\text { Romania }\end{array}$ & $\begin{array}{l}\text { Drying of } \\
\text { medicinal } \\
\text { plants }\end{array}$ & $\begin{array}{l}\text { Air } \\
\text { collector }\end{array}$ & \\
\hline China & $\begin{array}{l}\text { Hongxin } \\
\text { Aquaculture }\end{array}$ & $\begin{array}{l}\text { Water } \\
\text { heating }\end{array}$ & $\begin{array}{l}\text { Evacuated } \\
\text { tube } \\
\text { collector }\end{array}$ & \\
\hline USA & $\begin{array}{l}\text { Sonoma } \\
\text { Country } \\
\text { Herb } \\
\text { Exchange }\end{array}$ & $\begin{array}{l}\text { Drying of } \\
\text { herbs }\end{array}$ & $\begin{array}{l}\text { Air } \\
\text { collector }\end{array}$ & \\
\hline Mexico & $\begin{array}{l}\text { Buenavista } \\
\text { Greenhouse }\end{array}$ & $\begin{array}{l}\text { Space } \\
\text { heating }\end{array}$ & $\begin{array}{l}\text { Parallel } \\
\text { trough } \\
\text { collector }\end{array}$ & \\
\hline \multirow{2}{*}{ Germany } & Krimmer & $\begin{array}{l}\text { Drying of } \\
\text { wild flower } \\
\text { seeds }\end{array}$ & $\begin{array}{l}\text { Flat plate } \\
\text { collector }\end{array}$ & \\
\cline { 2 - 5 } & Woltow & $\begin{array}{l}\text { Water } \\
\text { heating }\end{array}$ & $\begin{array}{l}\text { Parallel } \\
\text { trough } \\
\text { collector }\end{array}$ & \\
\hline
\end{tabular}

Table 25: Existing SHIP in agricultural industries [101].

Rural industries research and Development Corporation worked on developing a solar drying machine for agricultural products [47]. Silva et al. worked on the solar process heat driven vegetable 
preservation industry by thermal treatment and canning in Spain [85]. Hussain et al. reviewed solar process heating systems used in agricultural industries [34].

\subsection{Manufacturing of Leather products}

Several Asian countries like Thailand, China, India and other countries are operating with solar process heating based leather production. Manufacturing industries of leather products principally involve the retanning process and water heating for solar industrial process heat utilization. The evacuatedttube collector is mostly suitable for these types of operations (Table 26).

\begin{tabular}{|l|l|l|l|l|}
\hline Country & Name & $\begin{array}{l}\text { Industrial } \\
\text { Operation }\end{array}$ & $\begin{array}{l}\text { Solar } \\
\text { Collector }\end{array}$ & Temperature $\left({ }^{\circ} \mathbf{C}\right)$ \\
\hline Thailand & $\begin{array}{l}\text { Ayuttaya } \\
\text { Tannery }\end{array}$ & Retanning process & $\begin{array}{l}\text { Evacuated } \\
\text { tube } \\
\text { collector }\end{array}$ & $\sim 80$ \\
\cline { 2 - 5 } & $\begin{array}{l}\text { Sadesa } \\
\text { Leather }\end{array}$ & Retanning process & $\begin{array}{l}\text { Evacuated } \\
\text { tube } \\
\text { collector }\end{array}$ & \\
\hline Austria & $\begin{array}{l}\text { Gerberei } \\
\text { Kolblinger }\end{array}$ & $\begin{array}{l}\text { Hot water for the } \\
\text { wet process of } \\
\text { leather retanning }\end{array}$ & $\begin{array}{l}\text { Flat plate } \\
\text { collector }\end{array}$ & \\
\hline China & $\begin{array}{l}\text { Heshan } \\
\text { Bestway } \\
\text { Leather }\end{array}$ & Retanning process & $\begin{array}{l}\text { Evacuated } \\
\text { tube } \\
\text { collector }\end{array}$ & $\sim 70$ \\
\hline India & Leo Leather & $\begin{array}{l}\text { Chemical mixing, } \\
\text { drying }\end{array}$ & $\begin{array}{l}\text { Flat plate } \\
\text { collector }\end{array}$ & \\
\hline Kenya & $\begin{array}{l}\text { Nairobi } \\
\text { Tannery }\end{array}$ & Retanning process & $\begin{array}{l}\text { Evacuated } \\
\text { tube } \\
\text { collector }\end{array}$ & \\
\hline Vietnam & $\begin{array}{l}\text { Saigan } \\
\text { Tantec }\end{array}$ & Retanning process & $\begin{array}{l}\text { Evacuated } \\
\text { tube } \\
\text { collector }\end{array}$ & $\sim 70$ \\
\hline Greece & $\begin{array}{l}\text { Tripau- } \\
\text { katsouris } \\
\text { leather } \\
\text { treatment } \\
\text { factory }\end{array}$ & Leather treatment & $\begin{array}{l}\text { Flat plate } \\
\text { collector }\end{array}$ & $48 \sim 84$ \\
& & & & \\
& & & \\
\hline
\end{tabular}

Table 26: Existing SHIP in leather industries [101].

\subsection{Fabricated Metal Industries}

In fabricated metal industries in mostly European countries solar process heat is used for water heating, process drying and cleaning processes, which are the most widely used solar heat driven processes. The maximum attained temperature is $180^{\circ} \mathrm{C}$ operated with various types of solar collectors (Table 27).

\begin{tabular}{|c|c|c|c|c|}
\hline Country & Name & $\begin{array}{l}\text { Industrial } \\
\text { Operation }\end{array}$ & $\begin{array}{l}\text { Solar } \\
\text { Collector }\end{array}$ & Temperature $\left({ }^{\circ} \mathrm{C}\right)$ \\
\hline \multirow[t]{2}{*}{ Germany } & Alanod Solar & $\begin{array}{l}\text { Production of } \\
\text { saturated } \\
\text { steam }\end{array}$ & $\begin{array}{l}\text { Parallel } \\
\text { trough } \\
\text { collector }\end{array}$ & $\sim 143$ \\
\hline & $\begin{array}{l}\text { Lackiercenter } \\
\text { Shulte }\end{array}$ & $\begin{array}{l}\text { Heating of } \\
\text { painting \& } \\
\text { drying } \\
\text { chamber }\end{array}$ & $\begin{array}{l}\text { Evacuated } \\
\text { tube } \\
\text { collector }\end{array}$ & \\
\hline Austria & Julius Blum & $\begin{array}{l}\text { Heating of } \\
\text { pre-treatment } \\
\text { washbasins } \\
\text { of the coating } \\
\text { device }\end{array}$ & $\begin{array}{l}\text { Evacuated } \\
\text { tube } \\
\text { collector }\end{array}$ & $\sim 85^{\star \star}$ \\
\hline Spain & Pincasa & Heat oven & $\begin{array}{l}\text { Other of } \\
\text { various } \\
\text { collectors }\end{array}$ & $\sim 180$ \\
\hline Portugal & Silampos S.A. & $\begin{array}{l}\text { Process } \\
\text { wash \& } \\
\text { drying } \\
\text { finished } \\
\text { product }\end{array}$ & $\begin{array}{l}\text { Parallel } \\
\text { trough } \\
\text { collector }\end{array}$ & $50 \sim 160$ \\
\hline India & $\begin{array}{l}\text { SKF } \\
\text { Technologies }\end{array}$ & $\begin{array}{l}\text { Heat the } \\
\text { water for }\end{array}$ & $\begin{array}{l}\text { Parallel } \\
\text { trough }\end{array}$ & $\sim 95$ \\
\hline
\end{tabular}

\begin{tabular}{|l|l|l|l|l|}
\hline & Mysore & circulation & collector & \\
\hline France & $\begin{array}{l}\text { Viessmann } \\
\text { Faulquemont }\end{array}$ & $\begin{array}{l}\text { Heating up } \\
\text { an alkaline } \\
\text { cleaning bath }\end{array}$ & $\begin{array}{l}\text { Evacuated } \\
\text { tube } \\
\text { collector }\end{array}$ & $\sim 60$ \\
\hline
\end{tabular}

Table 27: Existing SHIP in fabricated metal industries [101].

\subsection{Other Manufacturing Industries}

Different countries in Europe, south-east Asia and from Africa are using solar industrial process heat for their core industries like tobacco, wearing apparel, wood, plastic, manufacturing of electrical or mechanical equipment, furniture production units or different sorts of repair and installation houses. The most widely used industrial processes here are drying, washing, water heating, cooking etc. Exceptions include degreasing wheels, volcanizing cables and conditioning tobacco. Most of the processes operate within lowtemperature range applications using flat-plate collector or evacuatedtube collector (Table 28) [52,65,70,75,78].

\begin{tabular}{|c|c|c|c|c|c|}
\hline $\begin{array}{l}\text { Industry } \\
\text { Type }\end{array}$ & Country & Name & $\begin{array}{l}\text { Industrial } \\
\text { Operation }\end{array}$ & $\begin{array}{l}\text { Solar } \\
\text { Collect } \\
\text { or }\end{array}$ & $\begin{array}{l}\text { Tempera } \\
\text { ture }\left({ }^{\circ} \mathrm{C}\right)\end{array}$ \\
\hline \multirow[t]{2}{*}{ Tobacco } & Argentina & $\begin{array}{l}\text { Grammer } \\
\text { Solar } \\
\text { Argentina }\end{array}$ & $\begin{array}{l}\text { Drying of } \\
\text { tobacco }\end{array}$ & $\begin{array}{l}\text { Air } \\
\text { collector }\end{array}$ & \\
\hline & India & $\begin{array}{l}\text { India } \\
\text { Tobacco } \\
\text { Dlvision }\end{array}$ & $\begin{array}{l}\text { Conditioning } \\
\text { of tobacco }\end{array}$ & $\begin{array}{l}\text { Air } \\
\text { collector }\end{array}$ & $\sim 95$ \\
\hline \multirow[t]{2}{*}{$\begin{array}{l}\text { Wearing } \\
\text { Apparel }\end{array}$} & India & $\begin{array}{l}\text { Chelsea } \\
\text { Jeans }\end{array}$ & $\begin{array}{l}\text { Hot water } \\
\text { dyeing } \\
\text { process }\end{array}$ & $\begin{array}{l}\text { Flat } \\
\text { plate } \\
\text { collector }\end{array}$ & \\
\hline & Vietnam & $\begin{array}{l}\text { Saitex } \\
\text { Jeans }\end{array}$ & $\begin{array}{l}\text { Solar heat for } \\
\text { jeans washing } \\
\text { process }\end{array}$ & $\begin{array}{l}\text { Flat } \\
\text { plate } \\
\text { collector }\end{array}$ & \\
\hline Wood & Spain & $\begin{array}{l}\text { Instituto } \\
\text { del corcho }\end{array}$ & Cork cooking & $\begin{array}{l}\text { Other or } \\
\text { various } \\
\text { collector } \\
\mathrm{s}\end{array}$ & $\sim 110$ \\
\hline $\begin{array}{l}\text { Rubber \& } \\
\text { Plastic } \\
\text { Products }\end{array}$ & Thailand & $\begin{array}{l}\text { Inter } \\
\text { Rubber } \\
\text { Latex Co. } \\
\text { Ltd. }\end{array}$ & $\begin{array}{l}\text { Drying of } \\
\text { natural rubber }\end{array}$ & $\begin{array}{l}\text { Air } \\
\text { collector }\end{array}$ & \\
\hline \multirow[t]{2}{*}{$\begin{array}{l}\text { Concrete } \\
\text { Component } \\
\text { s }\end{array}$} & \multirow[t]{2}{*}{ Austria } & $\begin{array}{l}\text { Habau } \\
\text { Concrete } \\
\text { Componen } \\
t \\
\text { Production }\end{array}$ & $\begin{array}{l}\text { Heating of } \\
\text { formwork of } \\
\text { wooden } \\
\text { planks, drying } \\
\text { of concrete } \\
\text { components }\end{array}$ & $\begin{array}{l}\text { Flat } \\
\text { plate } \\
\text { collector }\end{array}$ & $16 \sim 25$ \\
\hline & & $\begin{array}{l}\text { Leitt Beton } \\
\text { Gmbh }\end{array}$ & $\begin{array}{l}\text { drying of pre- } \\
\text { fabricated } \\
\text { concrete } \\
\text { components }\end{array}$ & $\begin{array}{l}\text { Flat } \\
\text { plate } \\
\text { collector }\end{array}$ & \\
\hline $\begin{array}{l}\text { Computer, } \\
\text { electronic \& } \\
\text { optical } \\
\text { products }\end{array}$ & Sweden & $\begin{array}{l}\text { Bosmans } \\
\text { Lackering }\end{array}$ & $\begin{array}{l}\text { Hot water for } \\
\text { chemical } \\
\text { baths that } \\
\text { provide gold } \\
\text { plating for } \\
\text { radio cabinets }\end{array}$ & $\begin{array}{l}\text { Parallel } \\
\text { trough } \\
\text { collector }\end{array}$ & $\sim 160$ \\
\hline \multirow[t]{2}{*}{$\begin{array}{l}\text { Manufacturi } \\
\mathrm{ng} \text { of } \\
\text { Electrical } \\
\text { Equipment }\end{array}$} & \multirow[t]{2}{*}{ India } & $\begin{array}{l}\text { Bergen } \\
\text { Solar }\end{array}$ & $\begin{array}{l}\text { Integrated in } \\
\text { existing } \\
\text { processes }\end{array}$ & $\begin{array}{l}\text { Other or } \\
\text { various } \\
\text { collector } \\
\mathrm{s}\end{array}$ & $120 \sim 140$ \\
\hline & & $\begin{array}{l}\text { Hindusthan } \\
\text { Vidyut } \\
\text { Products }\end{array}$ & $\begin{array}{l}\text { Volcanizing of } \\
\text { cables }\end{array}$ & $\begin{array}{l}\text { Other or } \\
\text { various } \\
\text { collector } \\
\mathrm{s}\end{array}$ & $\sim 85$ \\
\hline \multirow[b]{3}{*}{$\begin{array}{l}\text { Manufacturi } \\
\text { ng of } \\
\text { Machinery }\end{array}$} & $\begin{array}{l}\text { South } \\
\text { Africa }\end{array}$ & $\begin{array}{l}\text { ABB } \\
\text { Longmead } \\
\text { ow }\end{array}$ & $\begin{array}{l}\text { Other process } \\
\text { heating }\end{array}$ & $\begin{array}{l}\text { Flat } \\
\text { plate } \\
\text { collector }\end{array}$ & \\
\hline & \multirow[t]{2}{*}{ Austria } & $\begin{array}{l}\text { Hoval } \\
\text { Marchtrenk }\end{array}$ & $\begin{array}{l}\text { Solar hot } \\
\text { water for } \\
\text { powder } \\
\text { coating }\end{array}$ & $\begin{array}{l}\text { Flat } \\
\text { plate } \\
\text { collector }\end{array}$ & \\
\hline & & $\begin{array}{l}\text { Kral Pump } \\
\text { Factory }\end{array}$ & $\begin{array}{l}\text { Hot water } \\
\text { space heating } \\
\& \text { cooling }\end{array}$ & $\begin{array}{l}\text { Evacuat } \\
\text { ed tube } \\
\text { collector }\end{array}$ & \\
\hline
\end{tabular}




\begin{tabular}{|c|c|c|c|c|c|}
\hline & India & $\begin{array}{l}\text { Wheels } \\
\text { India }\end{array}$ & $\begin{array}{l}\text { To degrease } \\
\text { the wheels } \\
\text { before the } \\
\text { final painting }\end{array}$ & $\begin{array}{l}\text { Evacuat } \\
\text { ed tube } \\
\text { collector }\end{array}$ & \\
\hline & $\begin{array}{l}\text { Switzerla } \\
\text { nd }\end{array}$ & $\begin{array}{l}\text { Zehnder } \\
\text { Group AG }\end{array}$ & $\begin{array}{l}\text { Heating the } \\
\text { pre-treatment } \\
\text { washbasins of } \\
\text { the coating } \\
\text { device }\end{array}$ & $\begin{array}{l}\text { Evacuat } \\
\text { ed tube } \\
\text { collector }\end{array}$ & $\sim 90$ \\
\hline $\begin{array}{l}\text { Transport } \\
\text { Equipment }\end{array}$ & India & $\begin{array}{l}\text { Harite } \\
\text { Seatings } \\
\text { Systems } \\
\text { Limited }\end{array}$ & $\begin{array}{l}\text { Cleaning } \\
\text { automobile } \\
\text { parts before } \\
\text { painting }\end{array}$ & $\begin{array}{l}\text { Evacuat } \\
\text { ed tube } \\
\text { collector }\end{array}$ & $55 \sim 60$ \\
\hline Furniture & Austria & $\begin{array}{l}\text { Carpenting } \\
\text { Hamminge } \\
r\end{array}$ & Wood Drying & $\begin{array}{l}\text { Flat } \\
\text { plate } \\
\text { collector }\end{array}$ & $25 \sim 115$ \\
\hline $\begin{array}{l}\text { Repairing \& } \\
\text { Installation }\end{array}$ & Germany & $\begin{array}{l}\text { Lackiererei } \\
\text { Vogel }\end{array}$ & $\begin{array}{l}\text { Heat cabins } \\
\text { from and } \\
\text { generate hot } \\
\text { air for drying } \\
\text { process }\end{array}$ & $\begin{array}{l}\text { Evacuat } \\
\text { ed tube } \\
\text { collector }\end{array}$ & $\begin{array}{l}22 \sim 24,60 \\
\sim 70\end{array}$ \\
\hline
\end{tabular}

Table 28: Existing SHIP in other different type manufacturing industries [101].

\section{Summary and Future Research Scope}

Table 29 summarizes the current literature reviews of solar industrial process heat applications in different aspects like integration at supply level or process level, process heat integration feasibilities based on simulation and cost analysis. But no such work gives an extensive survey on all industries based on their operations/processes where a solar process heating system is currently in use, based on their available thermal energy generation capabilities, including solar collector types and highest amount of process heating temperature that can be achieved through solar heat in that particular area. This paper will certainly be a valuable resource to the literature which summarizes the potential industrial sectors, potential process heating operations for creating a future zero-carbon emission industrial zone.

\begin{tabular}{|c|c|c|c|c|c|}
\hline $\begin{array}{l}\text { Literature } \\
\text { work }\end{array}$ & $\begin{array}{l}\text { Potential } \\
\text { industrial } \\
\text { sectors/ } \\
\text { process } \\
\text { identification }\end{array}$ & $\begin{array}{l}\text { Feasibility } \\
\text { analysis/ } \\
\text { Integration } \\
\text { problems }\end{array}$ & $\begin{array}{l}\text { Modelli } \\
\text { ng and } \\
\text { simulati } \\
\text { on }\end{array}$ & $\begin{array}{l}\text { Experime } \\
\text { ntal } \\
\text { investigati } \\
\text { on }\end{array}$ & $\begin{array}{l}\text { Solar } \\
\text { collecto } \\
\text { r type, } \\
\text { temper } \\
\text { ature } \\
\text { require } \\
\text { ments }\end{array}$ \\
\hline $\begin{array}{l}\text { Weiss et } \\
\text { Al.[94] }\end{array}$ & 3 key sectors & $\begin{array}{l}\text { Solution to } \\
\text { integration } \\
\text { problems }\end{array}$ & No & No & $\mathrm{N} / \mathrm{A}$ \\
\hline $\begin{array}{l}\text { Kalogirou[3 } \\
\text { 8] }\end{array}$ & No & No & $\begin{array}{l}\text { TRNSY } \\
\text { S } \\
\text { simulati } \\
\text { on }\end{array}$ & No & $\begin{array}{l}\text { Paraboli } \\
\text { c-trough } \\
\text { collector }\end{array}$ \\
\hline Esen[21] & No & No & No & $\begin{array}{l}\text { Yes, } \\
\text { based on } \\
\text { different } \\
\text { refrigerant } \\
\mathrm{s}\end{array}$ & $\begin{array}{l}\text { Vacuum } \\
\text {-tube } \\
\text { collector }\end{array}$ \\
\hline $\begin{array}{l}\text { Schnitzer } \\
\text { et Al.[77] }\end{array}$ & $\begin{array}{ll}5 & \text { key } \\
\text { sectors, } 8 & \text { key } \\
\text { processes } & \\
\end{array}$ & $\begin{array}{l}\text { System } \\
\text { integration } \\
\text { feasibilities }\end{array}$ & No & No & $\mathrm{N} / \mathrm{A}$ \\
\hline $\begin{array}{l}\text { IEA SHC } \\
\text { TASK } \\
33[36]\end{array}$ & 10 key sectors & No & $\mathrm{N} / \mathrm{A}$ & $\mathrm{N} / \mathrm{A}$ & $\mathrm{N} / \mathrm{A}$ \\
\hline $\begin{array}{l}\text { Mekhlief et } \\
\text { Al.[43] }\end{array}$ & $\begin{array}{ll}11 & \text { key } \\
\text { sectors, } 6 \text { key } \\
\text { processes }\end{array}$ & No & No & No & $\mathrm{N} / \mathrm{A}$ \\
\hline $\begin{array}{l}\text { Lauterbach } \\
\text { et Al.[44] }\end{array}$ & $\begin{array}{l}7 \text { key sectors, } \\
3 \text { key } \\
\text { processes }\end{array}$ & $\begin{array}{l}\text { Feasibility } \\
\text { and cost } \\
\text { analysis }\end{array}$ & $\begin{array}{l}\text { TRNSY } \\
\text { S } \\
\text { simulati } \\
\text { on }\end{array}$ & No & No \\
\hline $\begin{array}{l}\text { Lauterbach } \\
\text { et Al.[42] }\end{array}$ & No & No & Yes & Yes & $\mathrm{N} / \mathrm{A}$ \\
\hline $\begin{array}{l}\text { Pietruschk } \\
\text { a et Al.[69] }\end{array}$ & No & No & $\begin{array}{l}\text { Simulati } \\
\text { on for } \\
\text { control } \\
\text { and }\end{array}$ & No & $\begin{array}{l}3 \\
\text { different } \\
\text { collector } \\
\text { s }\end{array}$ \\
\hline
\end{tabular}

\begin{tabular}{|c|c|c|c|c|c|}
\hline & & & $\begin{array}{l}\text { optimiza } \\
\text { tion }\end{array}$ & & \\
\hline $\begin{array}{ll}\text { Vajen } & \text { et } \\
\text { Al.[91] } & \end{array}$ & 4 key sectors & $\begin{array}{l}\text { System } \\
\text { integration } \\
\text { feasibilities }\end{array}$ & No & No & No \\
\hline $\begin{array}{l}\text { Calderoni } \\
\text { et Al.[9] }\end{array}$ & No & $\begin{array}{l}\text { Feasibility } \\
\text { study and } \\
\text { economic } \\
\text { analysis }\end{array}$ & No & No & No \\
\hline $\begin{array}{l}\text { Montes et } \\
\text { Al.[56] }\end{array}$ & No & No & No & Yes & $\begin{array}{l}\text { Paraboli } \\
\text { c trough } \\
\text { collector }\end{array}$ \\
\hline $\begin{array}{l}\text { Frein et } \\
\text { Al.[27] }\end{array}$ & No & No & No & Yes & $\mathrm{N} / \mathrm{A}$ \\
\hline $\begin{array}{l}\text { Schramm } \\
\text { et Al.[78] }\end{array}$ & No & $\begin{array}{l}\text { Storage } \\
\text { systems }\end{array}$ & Yes & Yes & $\begin{array}{l}4 \\
\text { systems }\end{array}$ \\
\hline $\begin{array}{ll}\text { Silva } & \text { et } \\
\text { Al.[83] }\end{array}$ & No & $\begin{array}{l}\text { Economic } \\
\text { analysis and } \\
\text { optimization }\end{array}$ & No & No & $\begin{array}{l}\text { Paraboli } \\
\text { c trough } \\
\text { collector }\end{array}$ \\
\hline $\begin{array}{ll}\text { Silva } & \text { et } \\
\text { Al.[85] } & \end{array}$ & No & $\begin{array}{l}\text { Uncertainty } \\
\text { and } \\
\text { sensitivity } \\
\text { analysis }\end{array}$ & No & No & $\begin{array}{l}\text { Pataboli } \\
\text { c trough } \\
\text { collector }\end{array}$ \\
\hline $\begin{array}{l}\text { Larcher et } \\
\text { Al.[41] }\end{array}$ & No & No & No & Yes & $\begin{array}{l}\text { Paraboli } \\
\text { c trough } \\
\text { collector }\end{array}$ \\
\hline $\begin{array}{l}\text { Coccia et } \\
\text { Al.[11] }\end{array}$ & No & No & No & Yes & $\begin{array}{l}\text { Paraboli } \\
\text { c trough } \\
\text { collector }\end{array}$ \\
\hline Schmitt[65] & No & $\begin{array}{l}\text { System } \\
\text { integration } \\
\text { by load } \\
\text { profile }\end{array}$ & No & No & No \\
\hline $\begin{array}{l}\text { This } \\
\text { Research }\end{array}$ & $\begin{array}{l}11 \text { key } \\
\text { sectors, } 6 \\
\text { key } \\
\text { processes }\end{array}$ & No & No & No & Yes \\
\hline
\end{tabular}

Table 29: Comparison among the methodologies of the related works.

For sustainable energy development in future, the crucial factor is reducing the carbon di-oxide emission thus reducing the consumption of non-renewable fuels. To limit the utilization of fossil fuels in today's industrialized world, it is obviously necessary to involve renewable energy sources to supply both process heat and electricity in industries. Electricity generation from solar energy is already widely in use. But supplying heat energy to run industrial process operations is still very limited in operation. A major drawback for lack of implementing solar process heating systems is significant capital cost of installation- which is a barrier for small and medium scale industries. To get a clear overview about the efficiency of solar industrial process heating system- industrial process operations should be analyzed through simulation to see whether solar process heat can generate the required temperature to run the plant, throughout its working time per day or its lifetime. To prove the reduction in greenhouse gas emission and calculate the impact upon the future environment, industry-specific or industrial-process specific lifecycle assessment can be carried out which will help to understand and commercialize the solar process heating system for reducing amount of fossil fuel consumption. An industry-wise or industrial-operation specific lifecycle inventory model can be created based on inventory database for further impact assessment. Lifecycle costing analysis can also give indication to manufacturers and consumers whether installing a solar process heating system can help their business or not.

\section{Conclusion}

In this review paper, existing industrial processes are categorized for the identification of typical applications for solar process heat and also future opportunities to integrate solar heat in the heat supply level of an industrial company. Industrial process-heat systems are analyzed 
in this paper with respect to collector technologies and demand temperatures. These results are applicable to every country within the same industry type along with similar weather and economic conditions. Solar energy should be given a chance even if the costs may not be so favorable, because of the depleted oil reserves, and increasing oil prices.

\section{References}

[1] Absi Halabi M, Al-Qattan a., Al-Otaibi a. Application of solar energy in the oil industry-Current status and future prospects. Renew Sustain Energy Rev. 2015;43:296-314.

[2] Aiken E, Lacroix N, Bucher K. Development of a Solar Water Pasteurizer With Integral Heat Exchanger ( Spihx ) 2007.

[3] Analysis M. Solar Process Heat in the Food Industry - Holger Müller 2016.

[4] Author G, Haagen M, Development B, Gmbh IS. Solar Process Heat for Industrial Processes 2013:1-7.

[5] Baig MH, Surovtseva D, Halawa E. The Potential of Concentrated Solar Power for Remote Mine Sites in the Northern Territory, Australia 2015;2015.

[6] Benz N, Gut M, Beikircher T, Ruß W. Solar process heat with nonconcentrating collectors for food industry. 1999 ISES Sol World Congr 2000:131-6. doi:10.1016/B978-008043895-5/50193-4.

[7] Brunner C, Slawitsch B, Giannakopoulou K, Schnitzer H. Industrial Process Indicators and Heat Integration in Industries. Joanneum Res Graz, Österreich 2008.

[8] Buker MS, Riffat SB. Solar assisted heat pump systems for low temperature water heating applications: A systematic review. Renew Sustain Energy Rev 2016;55:399-413. doi:10.1016/j.rser.2015.10.157.

[9] Calderoni M, Aprile M, Moretta S, Aidonis A, Motta M. Solar thermal plants for industrial process heat in Tunisia: Economic feasibility analysis and ideas for a new policy. Energy Procedia 2012;30:1390-400. doi:10.1016/j.egypro.2012.11.153.

[10] Celuppi R, Scapinello J, Andrade FGD, Revello JHP, Magro JD. Solar Energy Use for Water Pre-Heating in Boilers of AgroIndustries 2014;34:451-60.

[11] Coccia G, Di Nicola G, Sotte M. Design, manufacture, and test of a prototype for a parabolic trough collector for industrial process heat.Renew Energy 2014;74:727-36. doi:10.1016/j.renene.2014.08.077.

[12] Cottret N, Menichetti E. Technical Study Report on SOLAR HEAT FOR INDUSTRIAL PROCESSES (SHIP) State of the art in the Mediterranean region 2010.

[13] Deliverable C. Potential studies on solar process heat worldwide 2015.

[14] Desai DD, Raol JB, Patel S, Chauhan I. Application of Solar energy for sustainable Dairy Development. Eur J Sustain Dev 2013;2:131-40.

[15] Dinter F, Harms TM. OPPORTUNITIES FOR CONCENTRATED SOLAR THERMAL HEAT IN THE MINERALS PROCESSING INDUSTRY n.d.

[16] Duffie J a., Beckman W a., Worek WM. Solar Engineering of Thermal Processes, 4nd ed. vol. 116. 2003.

[17] Eglinton T, Hinkley J, Beath A, Dell'Amico M. Potential applications of concentrated solar thermal technologies in the Australian minerals processing and extractive metallurgical industry. Jom 2013;65:1710-20. [12] Eswara AR, Ramakrishnarao M. Solar energy in food processing - a critical appraisal. J Food Sci Technol 2012;50:209-27.

[18] El-Sebaii AA, Shalaby SM. Solar drying of agricultural products: A review. Renew Sustain Energy Rev 2012;16:37-43. doi:10.1016/j.rser.2011.07.134.
[19] Esen H, Esen M, Ozsolak O. Modelling and experimental performance analysis of solar-assisted ground source heat pump system. J Exp Theor Artif Intell 2017;29:1-17. doi:10.1080/0952813X.2015.1056242.

[20] Esen M. Thermal performance of a solar-aided latent heat store used for space heating by heat pump. Sol Energy 2000;69:15-25. doi:10.1016/S0038-092X(00)00015-3.

[21] Esen M. Thermal performance of a solar cooker integrated vacuum-tube collector with heat pipes containing different refrigerants. Sol Energy 2004;76:751-7. doi:10.1016/j.solener.2003.12.009.

[22] Esen M, Esen H. Experimental investigation of a two-phase closed thermosyphon solar water heater. Sol Energy 2005;79:459-68. doi:10.1016/j.solener.2005.01.001.

[23] Esen M, Yuksel T. Experimental evaluation of using various renewable energy sources for heating a greenhouse. Energy Build 2013;65:340-51. doi:10.1016/j.enbuild.2013.06.018.

[24] Eswara AR, Ramakrishnarao M. Solar energy in food processing - a critical appraisal. J Food Sci Technol 2012;50:209_ 27. doi:10.1007/s13197-012-0739-3.

[25] European Solar Thermal Industry Federation. Solar Industrial Process Heat. State of the Art. Key Issues Renew Heat Eur 2006:1-15.

[26] Fbk LC, Fise PH. Scientific and Technological Alliance for Report on suitable Process heat and / or CHP systems for specific industries and integration guidelines 2014.

[27] Frein A, Calderoni M, Motta M. Solar thermal plant integration into an industrial process. Energy Procedia 2014;48:1152-63. doi:10.1016/j.egypro.2014.02.130.

[28] Fuller RJ. Solar Drying- A Technology for Sustainable Agriculture and Food Production. Sol Energy Convers Photoenergy Syst 2000;III:25.

[29] Gautam A, Chamoli S, Kumar A, Singh S. A review on technical improvements, economic feasibility and world scenario of solar water heating system. Renew Sustain Energy Rev 2017;68:54162. doi:10.1016/j.rser.2016.09.104.

[30] Frey P, Fischer S, Drück H, Jakob K. Monitoring Results of a Solar Process Heat System Installed at a Textile Company in Southern Germany. Energy Procedia 2015;70:615-20.

[31] Hassine I Ben, Helmke A, Heß S, Krummenacher P, Muster B, Schmitt B, et al. Solar process heat for production and advanced applications 2015:1-5.

[32] Hofm T. Brewing beer with solar heat 2011:1-4.

[33] Hubackova A, Kucerova I, Chrun R, Chaloupkova P, Banout J. Development of solar drying model for selected Cambodian fish species. Sci World J 2014;2014:10. doi:10.1155/2014/439431.

[34] Hussain MI, Lee GH. Utilization of Solar Energy in Agricultural Machinery Engineering: A Review. J Biosyst Eng 2015;40:186-92. doi:10.5307/JBE.2015.40.3.186.

[35] Ibrahim OM, Ghoneim AA, Halabi OA, Kamil A. Solar industrial process heat potential in Khartoum, Sudan. Sol Wind Technol 1990;7:649-54.

[36] IEA-ETSAP, IRENA. Solar heat for industrial processes Technology Brief 2015:37.

[37] Kabeel AE, Hamed MH, Omara ZM, Kandeal AW. Solar air heaters: Design configurations, improvement methods and applications - A detailed review. Renew Sustain Energy Rev 2016;70:1189-206. doi:10.1016/j.rser.2016.12.021.

[38] Kalogirou S. the Potential of Solar Energy in Food-Industry Process Heat Applications. J Chem Inf Model 2013;53:1689-99.

[39] Kalogirou SA. Parabolic trough collectors for industrial process heat in Cyprus. Energy 2002;27:813-30. doi:10.1016/S03605442(02)00018-X. 
[40] Kumar M, Sansaniwal SK, Khatak P. Progress in solar dryers for drying various commodities. Renew Sustain Energy Rev 2016;55:346-60. doi:10.1016/j.rser.2015.10.158.

[41] Larcher $M$, Rommel M, Bohren A, Frank E, Minder S. Characterization of a parabolic trough collector for process heat applications. Energy Procedia 2014;57:2804-11. doi:10.1016/j.egypro.2014.10.313.

[42] Lauterbach C, Schmitt B, Vajen K. System analysis of a lowtemperature solar process heat system. Sol Energy 2014;101:117-30. doi:10.1016/j.solener.2013.12.014.

[43] Lauterbach C, Schmitt B, Vajen K, Jordan U. Solar Process Heat in Breweries - Potential and Barriers of a New Application Area. Renew Energy 2009:645-7.

[44] Lauterbach C, Rad SJ, Schmitt B, Vajen K. Feasibility assessment of solar process heat applications. 30th ISES Bienn Sol World Congr 2011, SWC 2011 2011;4:3361-70.

[45] Lee GH. A Study for the Use of Solar Energy for Agricultural Industry - Solar Drying System Using Evacuated Tubular Solar Collector and Auxiliary Heater - 2013;38:41-7.

[46] Liu M, Wang S, Li K. Study of the Solar Energy Drying Device and Its Application in Traditional Chinese Medicine in Drying. Int $\mathrm{J}$ Clin Med 2015:271-80.

[47] Machine SD. Developing a Solar Drying Machine for Agricultural Products n.d.

[48] Martin Haagen; Christian Zahler; Elke Zimmermann; Mahmoud M. R. Al-Najami. Solar process steam for pharmaceutical industry in Jordan.pdf. Energy Procedia 2015;70:621-5.

[49] Mauthner F. Solar Heat for Industrial Applications 2014.

[50] Mauthner F, Hubmann M, Brunner C, Fink C. Manufacture of malt and beer with low temperature solar process heat. Energy Procedia 2014;48:1188-93.

[51] Mehrdadi N, Joshi SG, Nasrabadi T, Hoveidi H. Aplication of solar energy for drying of sludge from pharmaceutical industrial waste water and probable reuse. Int J Environ Res 2007;1:42-8.

[52] Meier A, Bonaldi E, Cella G, Lipinski W, Wuillemin D. Solar chemical reactor technology for industrial production of lime. Sol Energy 2006;80:1355-62. doi:10.1016/j.solener.2005.05.017.

[53] Mekhilef S, Saidur R, Safari A. A review on solar energy use in industries. Renew Sustain Energy Rev 2011;15:1777-90.

[54] Modi A, Buhler F, Andreasen JG, Haglind F. A review of solar energy based heat and power generation systems. Renew Sustain Energy Rev 2017;67:1047-64. doi:10.1016/j.rser.2016.09.075.

[55] Montero I, Miranda MT, Sepúlveda FJ, Arranz JI, Rojas CV, Nogales S. Solar dryer application for olive oil mill wastes. Energies 2015;8:14049-63. doi:10.3390/en81212415.

[56] Montes IEP, Benitez AM, Chavez OM, Herrera AEL. Design and Construction of a Parabolic Trough Solar Collector for Process Heat Production. Energy Procedia 2014;57:2149-58. doi:10.1016/j.egypro.2014.10.181.

[57] Morrison GL. Solar Water Heaters - Markets and New Developments n.d.

[58] Muneer T, Asif M, Cizmecioglu Z, Ozturk HK. Prospects for solar water heating within Turkish textile industry. Renew Sustain Energy Rev 2008;12:807-23.

[59] Muneer T, Maubleu S, Asif M. Prospects of solar water heating for textile industry in Pakistan. Renew Sustain Energy Rev 2006;10:1-23.

[60] Murray JP. Aluminum Production Using High-Temperature Solar Process Heat. Sol Energy 1999;66:133-42.

[61] Mustayen AGMB, Mekhilef S, Saidur R. Performance study of different solar dryers: A review. Renew Sustain Energy Rev 2014;34:463-70. doi:10.1016/j.rser.2014.03.020.

[62] Naik H, Baredar P, Kumar A. Medium temperature application of concentrated solar thermal technology: Indian perspective. Renew
Sustain Energy Rev 2017;76:369-78. doi:10.1016/j.rser.2017.03.014.

[63] Norton B. Industrial and agricultural applications of solar heat. Compr Renew Energy 2012;3:567-94.

[64] Norton B. Harnessing Solar Heat. Lect Notes Energy 2014;18:938.

[65] Omda E-A, Akoy M, Ayoub M, Ahmed EA. Design and Construction of A Solar Dryer for Mango Slices. Most 2004:1-7.

[66] Osama BM, Robert A. Solar Thermal Sterilization: A TRNSYS Performance Analysis 2012.

[67] Palaniappan C. Perspectives of solar food processing in India 2009:1-11.

[68] Paull RE, Hollyer J, Nakamura-tengan L, Shimabuku R, Paull RE. TITLE : Solar pasteurization of fruit and vegetable wash water. Report Submitted by : 2007:1-3.

[69] Pietruschka D, Fedrizzi R, Orioli F, Söll R, Stauss R. Demonstration of three large scale solar process heat applications with different solar thermal collector technologies. Energy Procedia 2012;30:755-64. doi:10.1016/j.egypro.2012.11.086.

[70] Pirasteh G, Saidur R, Rahman SMA, Rahim NA. A review on development of solar drying applications. Renew Sustain Energy Rev 2014;31:133-48. doi:10.1016/j.rser.2013.11.052.

[71] Pro A, Chie M, Pro C, Ac TR, Millenniu N. Renewable Energy for Industrial Applications 2015.

[72] Quijera JA, Alriols MG, Labidi J. Usage of solar energy in an industrial process. Chem Eng Trans 2011;25:875-80. doi:10.3303/CET1125146.

[73] Rabab Z, Hafiz A, Nasir A, Muhammad A, Zia ul H. Fabrication and performance study of a solar milk pasteurizer. Pakistan $\mathrm{J}$ Agric Sci 2009;46:162-8.

[74] Ramos C, Ramirez R, Beltran J. Potential assessment in Mexico for solar process heat applications in food and textile industries. Energy Procedia 2013;49:1879-84.

[75] Sarkar J, Bhattacharyya S. Operating characteristics of transcritical $\mathrm{CO} 2$ heat pump for simultaneous water cooling and heating. Arch Thermodyn 2012;33:23-40. doi:10.1002/er.

[76] Schmitt B. Classification of Industrial Heat Consumers for Integration of Solar Heat. Energy Procedia 2016;91:650-60.

[77] Schnitzer H, Brunner C, Gwehenberger G. Minimizing greenhouse gas emissions through the application of solar thermal energy in industrial processes. J Clean Prod 2007;15:1271-86.

[78] Schramm S, Adam M. Storage in solar process heat applications. Energy Procedia, vol. 48, 2014. doi:10.1016/j.egypro.2014.02.136.

[79] Sharma AK, Sharma C, Mullick SC, Kandpal TC. Potential of Solar Energy Utilization for Process Heating in Paper Industry in India: A Preliminary Assessment. vol. 79. Elsevier B.V.; 2015.

[80] Sharma AK, Sharma C, Mullick SC, Kandpal TC. Carbon mitigation potential of solar industrial process heating: Paper industry in India. J Clean Prod 2016;112:1683-91.

[81] Sharma AK, Sharma C, Mullick SC, Kandpal TC. Potential of solar industrial process heating in dairy industry in India and consequent carbon mitigation. J Clean Prod 2016.

[82] Shukla A, Singh R, Shukla P. Energy Sustainability Through Green Energy. Green Energy Technol 2015;201:147-62.

[83] Silva R, Berenguel M, Pérez M, Fernández-Garcia A. Thermoeconomic design optimization of parabolic trough solar plants for industrial process heat applications with memetic algorithms. Appl Energy 2014;113:603-14. doi:10.1016/j.apenergy.2013.08.017.

[84] Silva R, Pérez M, Berenguel M, Valenzuela L, Zarza E. Uncertainty and global sensitivity analysis in the design of parabolic-trough direct steam generation plants for process heat applications. Appl Energy 2014;121:233-44. doi:10.1016/j.apenergy.2014.01.095.

[85] Silva R, Cabrera FJ, Pérez-García M. Process heat generation with parabolic trough collectors for a vegetables preservation 
industry in Southern Spain. Energy Procedia 2014;48:1210-6. doi:10.1016/j.egypro.2014.02.137.

[86] States U. Solar Thermal Energy for Industrial Uses 2011.

[87] Stefan Heß. Solar Process Heat Generation: Guide to Solar Thermal System Design for Selected Industrial Processes 2011.

[88] Stewart M, Petrie J. A process systems approach to life cycle inventories for minerals: South African and Australian case studies. J Clean Prod 2006;14:1042-56.

[89] Taibi E, Gielen D, Bazilian M. The potential for renewable energy in industrial applications. Renew Sustain Energy Rev 2012;16:735-44.

[90] Uppal A, Kesari JP. Solar Industrial Process Heat in Indian Automobile Industry 2015;IV:117-23.

[91] Vajen K. Solar heat for industrial processes-Potential, technologies and applications. Int ... 2012:22-3.

[92] Vannoni C, Battisti R, Drigo S. Potential for solar heat in indutrial processes. IEA SHC Task 33 and and SolarPACES Task IV: Solar heat for industrial processes. lea 2008:1-21.

[93] Valenzuela L, Hernández-Lobón D, Zarza E. Sensitivity analysis of saturated steam production in parabolic trough collectors. Energy Procedia 2012;30:765-74. doi:10.1016/j.egypro.2012.11.087.

[94] Weiss W, Schweiger H, Battisti R. Market potential and system designs for industrial solar heat applications n.d.:2-7.

[95] Yuan G, Hong L, Li X, Xu L, Tang W, Wang Z. Experimental Investigation of a Solar Dryer System for Drying Carpet. Energy Procedia 2015;70:626-33. doi:10.1016/j.egypro.2015.02.170.

[96] Zahler C, Iglauer O. Solar process heat for sustainable automobile manufacturing. Energy Procedia 2012;30:775-82.

[97] OME report for GSWH-UNEP-UNDP 122012.

[98] OME report for GSWH-UNEP-UNDP 122012.

[99]http://iipdigital.usembassy.gov/st/english/texttrans/2007/06/200706 08150209eaifas0.9326593.htm|\#axzz4OcUvAnmP

[100] http://www.global-greenhouse-warming.com/sustainable-energyinvestment.html

[101] http://ship-plants.info/solar-thermal-plants

[102] https://www.euroheat.org/ 